Supporting Information

A Practical Improvement, Enhancing the Large-Scale Synthesis of (+)-Discodermolide:

\title{
A Third-Generation Approach
}

Amos B. Smith, III, ${ }^{\star}$ B. Scott Freeze, Ignacio Brouard, and Tomoyasu Hirose

Department of Chemistry, University of Pennsylvania, Philadelphia, PA 19104, U.S.A. 
Materials and Methods. Reactions were carried out in oven or flame-dried glassware under an argon atmosphere, unless otherwise noted. All solvents were reagent grade. Diethyl ether $\left(\mathrm{Et}_{2} \mathrm{O}\right)$ and tetrahydrofuran (THF) were freshly distilled from sodium/benzophenone under argon. $n$-Butyllithium and $t$-butyllithium were purchased from Aldrich. Reactions were magnetically stirred and monitored by thin layer chromotography (TLC) with $0.25 \mathrm{~mm}$ E. Merck pre-coated silica gel plates. Flash chromatography was performed with silica gel 60 (particle size $0.040-0.062 \mathrm{~mm}$ ) supplied by Silicycle and Sorbent Technologies. Yields refer to chromatographically and spectroscopically pure compounds, unless otherwise stated. Infrared spectra were recorded on a Jasco Model FT/IR-480 Plus spectrometer. Proton and carbon-13 NMR spectra were recorded on a Bruker AMX-500 spectrometer. Chemical shifts are reported relative to either chloroform $(\square 7.26)$ or benzene $(\square 7.15)$ for ${ }^{1} \mathrm{H}-\mathrm{NMR}$ and either chloroform ( $\square 77.0)$ or benzene $(\square 128.0)$ for ${ }^{13} \mathrm{C}$ NMR. Optical rotations were measured on a Perkin-Elmer model 241 polarimeter. High resolution mass spectra were measured at the University of Pennsylvania Mass Spectrometry Service Center.

\section{Model System}

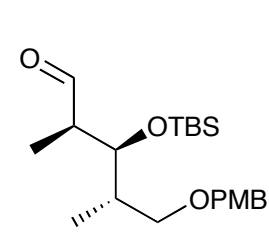

$(-)-17$

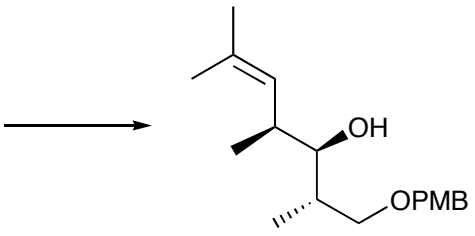

$(-)-19$

Alcohol (-)-19 Under argon, a solution of isopropyltriphenylphosphonium iodide $(4.41 \mathrm{~g}, 10.2 \mathrm{mmol})$, in toluene $(40 \mathrm{~mL})$, was treated with 0.6 M NaHMDS in toluene $(16.5 \mathrm{~mL}, 9.93 \mathrm{mmol}$ ) over $10 \mathrm{~min}$. at room temperature. After an additional $15 \mathrm{~min}$., the mixture was cooled to $-78{ }^{\circ} \mathrm{C}$. A solution of known aldehyde $(-)-17(2.62 \mathrm{~g}, 6.89 \mathrm{mmol})$ in toluene $(29 \mathrm{~mL})$ was added dropwise via cannula to the ylide solution at -78 ${ }^{\circ} \mathrm{C}$. After $5 \mathrm{~min}$., the resulting solution was warmed to room temperature and stirred for $25 \mathrm{~min}$. further. The reaction was quenched with $\mathrm{MeOH}(2 \mathrm{~mL})$, filtered, and concentrated to afford the crude trisubstituted olefin 18 (not shown), which was taken on without purification.

To a solution of the crude olefin 18 in $\mathrm{MeOH}(138 \mathrm{~mL})$ was added $3 \mathrm{~N} \mathrm{HCl}$ aq. solution $(27.6 \mathrm{~mL})$ over 4 $\mathrm{h}$ at room temperature. The mixture was stirred for $9 \mathrm{~h}$, whereupon the reaction mixture was neutralized with solid $\mathrm{NaHCO}_{3}$. $\mathrm{MeOH}$ was removed in vacuo, and the remaining mixture was diluted with $\mathrm{Et}_{2} \mathrm{O}$ (50 $\mathrm{mL}$ ), washed with $\mathrm{H}_{2} \mathrm{O}$, dried over $\mathrm{Na}_{2} \mathrm{SO}_{4}$, filtered, and concentrated. Flash chromatography (hexanes/EtOAc $=50 / 1 \square 3 / 1)$ afforded alcohol $(-)-19(1.10 \mathrm{~g}, 55 \%)$ as a colorless oil: $[\square]_{D}^{20}-2.5(c 1.0$, $\mathrm{CHCl}_{3}$ ); IR ( $\left.\mathrm{CHCl}_{3}\right) 3495$ (brs), 2962 (s), 2930 (s), 2867 (s), 1613 (s), 1586 (m), 1514 (s), 1456 (s), 1363 (m), $1302(\mathrm{~m}), 1249$ (s), $1173(\mathrm{~m}), 1084(\mathrm{~s}), 1037(\mathrm{~s}), 986(\mathrm{~m}), 821(\mathrm{~m}) \mathrm{cm}^{-1} ;{ }^{1} \mathrm{H}-\mathrm{NMR}\left(500 \mathrm{MHz}, \mathrm{CDCl}_{3}\right) \square$ $7.24(\mathrm{~d}, J=8.4 \mathrm{~Hz}, 2 \mathrm{H}), 6.87(\mathrm{~d}, J=8.4 \mathrm{~Hz}, 2 \mathrm{H}), 5.06(\mathrm{~d}, J=9.5 \mathrm{~Hz}, 1 \mathrm{H}), 4.45(\mathrm{~d}, J=11.5 \mathrm{~Hz}, 1 \mathrm{H}), 4.41$ (d, $J=11.5 \mathrm{~Hz}, 1 \mathrm{H}), 3.80(\mathrm{~s}, 3 \mathrm{H}), 3.58(\mathrm{dd}, J=9.3,4.0 \mathrm{~Hz}, 1 \mathrm{H}), 3.41(\mathrm{dd}, J=9.3,5.5 \mathrm{~Hz}, 1 \mathrm{H}), 3.28$ 
(apparent t, $J=6.0 \mathrm{~Hz}, 1 \mathrm{H}), 3.12(\mathrm{bs}, 1 \mathrm{H}), 2.45(\mathrm{~m}, 1 \mathrm{H}), 1.88(\mathrm{~m}, 1 \mathrm{H}), 1.68(\mathrm{~s}, 3 \mathrm{H}), 1.56(\mathrm{~s}, 3 \mathrm{H}), 0.99(\mathrm{~d}$, $J=7.1 \mathrm{~Hz}, 3 \mathrm{H}$ ), 0.97 (d, $J=6.7 \mathrm{~Hz}, 3 \mathrm{H}) ;{ }^{13} \mathrm{C}-\mathrm{NMR}\left(125 \mathrm{MHz}, \mathrm{CDCl}_{3}\right) \square 159.3,130.3,129.9,129.3(2 \mathrm{C})$, 128.3, $113.8(2 \mathrm{C}), 80.1,74.1,73.2,55.2,36.1,35.6,25.8,17.8,15.4,14.9$; high resolution mass spectrum $\left(\right.$ ES $\left.^{+}\right) \mathrm{m} / \mathrm{z} 315.1945\left[(\mathrm{M}+\mathrm{Na})^{+}\right.$; calcd for $\left.\mathrm{C}_{18} \mathrm{H}_{28} \mathrm{O}_{3} \mathrm{Na}: 315.1936\right]$.

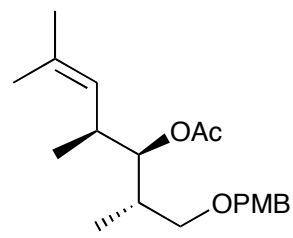

20

Acetate ester 20. To a solution of alcohol (-)-19 $(89.5 \mathrm{mg}, 0.307 \mathrm{mmol})$ and DMAP $(3.7 \mathrm{mg}, 0.0307$ $\mathrm{mmol})$ in pyridine $(0.77 \mathrm{~mL})$ was added acetyl chloride $(54.7 \mathrm{LL}, 0.766 \mathrm{mmol})$ at room temperature. The mixture was stirred for $1.5 \mathrm{~h}$, then diluted with a hexanes•EtOAc (2:1) solution $(10 \mathrm{~mL})$, and washed with saturated $\mathrm{NH}_{4} \mathrm{Cl}$ aq. and brine. The organic layer was dried over $\mathrm{Na}_{2} \mathrm{SO}_{4}$, filtered, and concentrated. Preparative TLC (hexanes/EtOAc $=4 / 1)$ afforded acetate $20(83.2 \mathrm{mg}, 81 \%)$ as a colorless oil: ${ }^{1} \mathrm{H}-\mathrm{NMR}$ $\left(500 \mathrm{MHz}, \mathrm{CDCl}_{3}\right) \square 7.24(\mathrm{~d}, J=8.6 \mathrm{~Hz}, 2 \mathrm{H}), 6.87$ (d, J = 8.6 Hz, 2H), 4.89 (ddd, J = 9.6, 1.3, $1.3 \mathrm{~Hz}, 1 \mathrm{H}$ ), 4.76 (dd, $J=7.8,4.8 \mathrm{~Hz}, 1 \mathrm{H}), 4.39$ (s, 2H), 3.80 (s, 3H), 3.49 (dd, $J=9.2,4.5 \mathrm{~Hz}, 1 \mathrm{H}$ ), 3.19 (apparent t, $J$ $=9.2 \mathrm{~Hz}, 1 \mathrm{H}), 2.67(\mathrm{~m}, 1 \mathrm{H}), 2.08(\mathrm{~m}, 1 \mathrm{H}), 2.03(\mathrm{~s}, 3 \mathrm{H}), 1.66(\mathrm{~d}, J=1.3 \mathrm{~Hz}, 3 \mathrm{H}), 1.57(\mathrm{~d}, J=1.3 \mathrm{~Hz}, 3 \mathrm{H})$, $0.96(\mathrm{~d}, J=6.9 \mathrm{~Hz}, 3 \mathrm{H}), 0.87(\mathrm{~d}, J=6.7 \mathrm{~Hz}, 3 \mathrm{H})$.

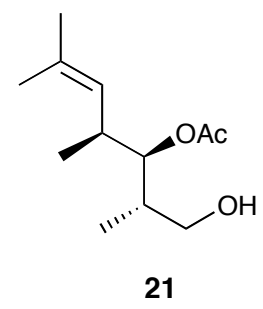

Alcohol 21. At $0{ }^{\circ} \mathrm{C}$, a solution of $20(82.2 \mathrm{mg}, 0.246 \mathrm{mmol})$ in $\mathrm{CH}_{2} \mathrm{Cl}_{2}(2.46 \mathrm{~mL})$ was treated with $\mathrm{H}_{2} \mathrm{O}$ $(0.098 \mathrm{~mL})$ and DDQ $(61.5 \mathrm{mg}, 0.271 \mathrm{mmol})$ and stirred for $1 \mathrm{~h} 20 \mathrm{~min}$. The reaction was quenched with saturated $\mathrm{NaHCO}_{3}$ aq. $(4 \mathrm{~mL})$ and the layers separated. The aqueous layer was then extracted with $\mathrm{CH}_{2} \mathrm{Cl}_{2}(2 \times 10 \mathrm{~mL})$. The combined organic layers were dried over $\mathrm{Na}_{2} \mathrm{SO}_{4}$, filtered, and concentrated. Flash chromatography (hexanes/EtOAc $=20 / 1 \square 1 / 1)$ provided primary alcohol $21(52.2 \mathrm{mg}, 99 \%)$ as a colorless oil): (Acyl migration precluded ${ }^{1} \mathrm{H}-\mathrm{NMR}$ on this substrate). 


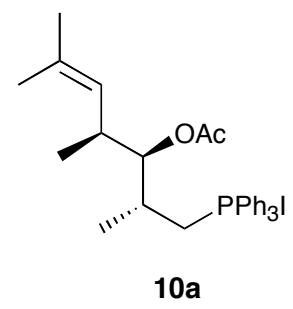

Wittig salt 10a. A solution of $21(50.5 \mathrm{mg}, 0.236 \mathrm{mmol}), \mathrm{PPh}_{3}(92.8 \mathrm{mg}, 0.354 \mathrm{mmol})$ and imidazole $(24.1 \mathrm{mg}, 0.354 \mathrm{mmol})$ in benzene $\cdot \mathrm{Et}_{2} \mathrm{O}(1: 2)(2.6 \mathrm{~mL})$ was cooled to $0{ }^{\circ} \mathrm{C}$ and treated with iodine (89.8 $\mathrm{mg}, 0.354 \mathrm{mmol})$. The reaction mixture was warmed to room temperature and stirred for $3.5 \mathrm{~h}$. The reaction was then quenched with saturated $\mathrm{NaHCO}_{3}$ aq. $(5 \mathrm{~mL})$. The layers were separated, and the aqueous layer was extracted with $\mathrm{Et}_{2} \mathrm{O}(3 \times 10 \mathrm{~mL})$. The combined organics were dried over $\mathrm{Na}_{2} \mathrm{SO}_{4}$, filtered, and concentrated. Flash chromatography (hexanes/EtOAc $=30 / 1$ ) provided a mixture of the desired alkyl iodide 9a and $\mathrm{PPh}_{3}$, which was taken onto the next step without further purification.

To the neat alkyl iodide 9a (mixed with $\mathrm{PPh}_{3}$ ) was added additional $\mathrm{PPh}_{3}(619 \mathrm{mg}, 2.36 \mathrm{mmol}$ ) and $i$ $\mathrm{Pr}_{2} \mathrm{NEt}(0.37 \mathrm{~mL})$ at room temperature. The mixture was warmed to $100{ }^{\circ} \mathrm{C}$ and stirred for $17 \mathrm{~h}$. The reaction mixture was cooled to room temperature and diluted with $\mathrm{CH}_{2} \mathrm{Cl}_{2}(0.5 \mathrm{~mL})$. The resulting solution was directly purified via flash chromatography $\left(\mathrm{CH}_{2} \mathrm{Cl}_{2} / \mathrm{CH}_{3} \mathrm{CN}=5 / 1 \square 2 / 1\right)$ to provide Wittig salt 10a (80.9 $\mathrm{mg}, 59 \%$ for 2 steps) and the mixture of cyclopentane byproducts ( $18.9 \mathrm{mg}, 41 \%$ for 2 steps).

Wittig salt (10a); light yellow oil; ${ }^{1} \mathrm{H}-\mathrm{NMR}\left(500 \mathrm{MHz}, \mathrm{C}_{6} \mathrm{D}_{6}\right) \square 7.84(\mathrm{~m}, 6 \mathrm{H}), 7.07(\mathrm{~m}, 9 \mathrm{H}), 5.38(\mathrm{~m}, 1 \mathrm{H})$, $4.95(\mathrm{~d}, J=9.4 \mathrm{~Hz}, 1 \mathrm{H}), 4.62(\mathrm{~d}, J=9.8 \mathrm{~Hz}, 1 \mathrm{H}), 3.26$ (apparent $\mathrm{t}, J=15.3 \mathrm{~Hz}, 1 \mathrm{H}), 2.74(\mathrm{~m}, 1 \mathrm{H}), 2.48$ (s, $3 \mathrm{H}), 1.88(\mathrm{~m}, 1 \mathrm{H}), 1.32(\mathrm{~s}, 3 \mathrm{H}), 1.26(\mathrm{~s}, 3 \mathrm{H}), 0.98(\mathrm{~d}, J=7.0 \mathrm{~Hz}, 3 \mathrm{H}), 0.95(\mathrm{~d}, J=6.5 \mathrm{~Hz}, 3 \mathrm{H})$; high resolution mass spectrum $\left(E S^{+}\right) \mathrm{m} / z$ 459.2437 [(M-I) ${ }^{+}$; calcd for $\mathrm{C}_{30} \mathrm{H}_{36} \mathrm{O} \mathrm{O}_{2} \mathrm{P}: 459.2453$ ].

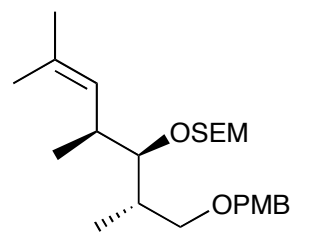

22

SEM ether 22. To a solution of alcohol (-)-19 (87.3 $\mathrm{mg}, 0.300 \mathrm{mmol})$ and $i-\operatorname{Pr}_{2} \mathrm{NEt}(260 \mathrm{LL}, 1.49 \mathrm{mmol})$ in $\mathrm{CH}_{2} \mathrm{Cl}_{2}(1.0 \mathrm{~mL})$ was added SEMCl $(159 \mathrm{LL}, 0.897 \mathrm{mmol})$ at room temperature. After stirring for $19.5 \mathrm{~h}$, the reaction was quenched with saturated $\mathrm{NH}_{4} \mathrm{Cl}$ aq. $(5 \mathrm{~mL})$ and extracted with $\mathrm{CHCl}_{3}(2 \times 10 \mathrm{~mL})$. The combined organic layers were dried over $\mathrm{Na}_{2} \mathrm{SO}_{4}$, filtered, and concentrated. Preparative TLC (hexanes/EtOAc $=6 / 1)$ provided SEM ether $22(112.5 \mathrm{mg}, 89 \%)$ as a colorless oil: ${ }^{1} \mathrm{H}-\mathrm{NMR}(500 \mathrm{MHz}$, $\left.\mathrm{CDCl}_{3}\right) \square 7.24(\mathrm{~d}, J=8.7 \mathrm{~Hz}, 2 \mathrm{H}), 6.86(\mathrm{~d}, J=8.7 \mathrm{~Hz}, 2 \mathrm{H}), 4.97(\mathrm{ddd}, J=9.6,1.3,1.3 \mathrm{~Hz}, 1 \mathrm{H}), 4.69(\mathrm{~d}, J$ 
$=6.7 \mathrm{~Hz}, 1 \mathrm{H}), 4.65(\mathrm{~d}, J=6.7 \mathrm{~Hz}, 1 \mathrm{H}), 4.40(\mathrm{~s}, 2 \mathrm{H}), 3.80(\mathrm{~s}, 3 \mathrm{H}), 3.65$ (apparent t, $J=8.5 \mathrm{~Hz}, 1 \mathrm{H}), 3.63$ (apparent t, $J=8.5 \mathrm{~Hz}, 1 \mathrm{H}), 3.54(\mathrm{dd}, J=9.1,4.3 \mathrm{~Hz}, 1 \mathrm{H}), 3.29(\mathrm{dd}, J=9.1,8.0 \mathrm{~Hz}, 1 \mathrm{H}), 3.24(\mathrm{dd}, J=$ 6.5, $5.0 \mathrm{~Hz}, 1 \mathrm{H}), 2.59(\mathrm{~m}, 1 \mathrm{H}), 2.02(\mathrm{~m}, 1 \mathrm{H}), 1.66(\mathrm{~d}, J=1.3 \mathrm{~Hz}, 3 \mathrm{H}), 1.57(\mathrm{~d}, J=1.3 \mathrm{~Hz}, 3 \mathrm{H}), 1.01(\mathrm{~d}, J=$ $6.9 \mathrm{~Hz}, 3 \mathrm{H}$ ), 0.94 (apparent t, $J=8.5 \mathrm{~Hz}, 2 \mathrm{H}), 0.94(\mathrm{~d}, J=6.7 \mathrm{~Hz}, 3 \mathrm{H}), 0.02(\mathrm{~s}, 9 \mathrm{H})$.

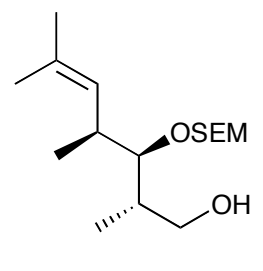

23

Alcohol 23. At $0{ }^{\circ} \mathrm{C}$, a solution of $22(110.2 \mathrm{mg}, 0.261 \mathrm{mmol})$ in $\mathrm{CH}_{2} \mathrm{Cl}_{2}(2.75 \mathrm{~mL})$ was treated with $\mathrm{H}_{2} \mathrm{O}$ $(0.10 \mathrm{~mL})$ and $\mathrm{DDQ}(65.2 \mathrm{mg}, 0.287 \mathrm{mmol})$, and stirred for $1 \mathrm{~h} 20 \mathrm{~min}$. The reaction was quenched with saturated $\mathrm{NaHCO}_{3}$ aq. (4 $\mathrm{mL}$ ) and the layers separated. The aqueous layer was then extracted with $\mathrm{CH}_{2} \mathrm{Cl}_{2}(2 \times 10 \mathrm{~mL})$. The combined organic layers were dried over $\mathrm{Na}_{2} \mathrm{SO}_{4}$, filtered, and concentrated. Flash chromatography (hexanes/EtOAc $=20 / 1 \square 1 / 1)$ provided primary alcohol $23(71.6 \mathrm{mg}, 91 \%)$ as a colorless oil): ${ }^{1} \mathrm{H}-\mathrm{NMR}\left(500 \mathrm{MHz}, \mathrm{CDCl}_{3}\right) \square 5.00$ (ddd, $\left.J=9.5,1.3,1.3 \mathrm{~Hz}, 1 \mathrm{H}\right), 4.72(\mathrm{~d}, J=6.7 \mathrm{~Hz}, 1 \mathrm{H}$ ), $4.68(\mathrm{~d}, J=6.7 \mathrm{~Hz}, 1 \mathrm{H}), 3.84(\mathrm{dd}, J=11.2,3.5 \mathrm{~Hz}, 1 \mathrm{H}), 3.75(\mathrm{dt}, J=9.7,8.2 \mathrm{~Hz}, 1 \mathrm{H}), 3.57(\mathrm{dt}, J=9.7$, $8.2 \mathrm{~Hz}, 1 \mathrm{H}), 3.48(\mathrm{dd}, J=11.2,4.0 \mathrm{~Hz}, 1 \mathrm{H}), 3.31(\mathrm{dd}, J=7.1,4.9 \mathrm{~Hz}, 1 \mathrm{H}), 2.63(\mathrm{~m}, 1 \mathrm{H}), 1.79(\mathrm{~m}, 1 \mathrm{H})$, $1.69(\mathrm{~d}, J=1.3 \mathrm{~Hz}, 3 \mathrm{H}), 1.62(\mathrm{~d}, J=1.3 \mathrm{~Hz}, 3 \mathrm{H}), 1.03(\mathrm{~d}, J=7.1 \mathrm{~Hz}, 3 \mathrm{H}), 0.96(\mathrm{dd}, J=8.2,8.2 \mathrm{~Hz}, 2 \mathrm{H})$, $0.95(\mathrm{~d}, J=6.8 \mathrm{~Hz}, 3 \mathrm{H}), 0.02(\mathrm{~s}, 9 \mathrm{H})$.

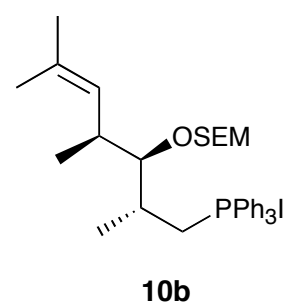

Wittig salt 10b. A solution of $23(71.6 \mathrm{mg}, 0.237 \mathrm{mmol}), \mathrm{PPh}_{3}(93.3 \mathrm{mg}, 0.356 \mathrm{mmol})$ and imidazole (24.2 $\mathrm{mg}, 0.356 \mathrm{mmol})$ in benzene $\cdot \mathrm{Et}_{2} \mathrm{O}(1: 2)(2.4 \mathrm{~mL})$ was cooled to $0{ }^{\circ} \mathrm{C}$, and treated with iodine $(90.3$ $\mathrm{mg}, 0.356 \mathrm{mmol})$. The reaction mixture was warmed to room temperature and stirred for $3.5 \mathrm{~h}$. The reaction was then quenched with saturated $\mathrm{NaHCO}_{3}$ aq. $(5 \mathrm{~mL})$. The mixture was extracted with $\mathrm{Et}_{2} \mathrm{O}(3 \mathrm{x}$ $10 \mathrm{~mL}$ ), and the combined organics were dried over $\mathrm{Na}_{2} \mathrm{SO}_{4}$, filtered, and concentrated. Flash chromatography (hexanes/EtOAc $=30 / 1$ ) provided a mixture of the desired alkyl iodide $9 \mathrm{~b}$ and $\mathrm{PPh}_{3}$, which was taken onto the next step without further purification.

To the neat alkyl iodide9b (mixed with $\mathrm{PPh}_{3}$ ) was added additional $\mathrm{PPh}_{3}(622 \mathrm{mg}, 2.37 \mathrm{mmol}$ ) and $i$ $\operatorname{Pr}_{2} \mathrm{NEt}(0.34 \mathrm{~mL})$ at room temperature. The mixture was warmed to $100{ }^{\circ} \mathrm{C}$ and stirred for $17 \mathrm{~h}$. The reaction mixture was cooled to room temperature and diluted with $\mathrm{CH}_{2} \mathrm{Cl}_{2}(0.5 \mathrm{~mL})$. The resulting solution 
was directly purified via flash chromatography $\left(\mathrm{CH}_{2} \mathrm{Cl}_{2} / \mathrm{CH}_{3} \mathrm{CN}=5 / 1 \square\right.$ 2/1) to give Wittig salt $10 \mathrm{~b}$ (100.0 $\mathrm{mg}, 63 \%$ for 2 steps) and the mixture of cyclopentane byproducts ( $23 \mathrm{mg}, 34 \%$ for 2 steps). Wittig salt 10b, light yellow oil; ' ${ }^{1} \mathrm{H}-\mathrm{NMR}\left(500 \mathrm{MHz}, \mathrm{C}_{6} \mathrm{D}_{6}\right) \square 7.91(\mathrm{~m}, 6 \mathrm{H}), 7.12(\mathrm{~m}, 9 \mathrm{H}), 5.39(\mathrm{~d}, J=9.1 \mathrm{~Hz}, 1 \mathrm{H})$, $5.19(\mathrm{~m}, 1 \mathrm{H}), 4.98(\mathrm{~d}, J=6.0 \mathrm{~Hz}, 1 \mathrm{H}), 4.94(\mathrm{~d}, J=6.0 \mathrm{~Hz}, 1 \mathrm{H}), 4.30$ (apparent t, $J=15.0 \mathrm{~Hz}, 1 \mathrm{H}), 4.07$ (dd, $J=6.4,4.9 \mathrm{~Hz}, 1 \mathrm{H}), 3.79(\mathrm{~m}, 1 \mathrm{H}), 3.71(\mathrm{~m}, 1 \mathrm{H}), 2.74(\mathrm{~m}, 1 \mathrm{H}), 2.14(\mathrm{~m}, 1 \mathrm{H}), 1.67(\mathrm{~s}, 3 \mathrm{H}), 1.61(\mathrm{~s}$, $3 \mathrm{H}), 1.03(\mathrm{~m}, 1 \mathrm{H}), 1.02(\mathrm{~d}, J=6.7 \mathrm{~Hz}, 3 \mathrm{H}), 0.98(\mathrm{~d}, J=6.8 \mathrm{~Hz}, 3 \mathrm{H}), 0.76(\mathrm{~m}, 1 \mathrm{H}),-0.01(\mathrm{~s}, 9 \mathrm{H})$; high resolution mass spectrum $\left(\mathrm{ES}^{+}\right) \mathrm{m} / \mathrm{z} 547.3141$ [(M-I) ${ }^{+}$; calcd for $\mathrm{C}_{34} \mathrm{H}_{48} \mathrm{O}_{2} \mathrm{PSi}$ : 547.3161].

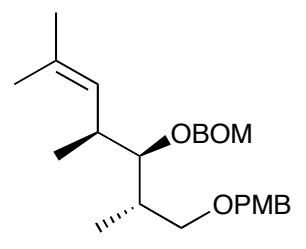

24

BOM ether 24. To a solution of alcohol (-)- 19 (97.7 mg, $0.335 \mathrm{mmol})$ and $i-\operatorname{Pr}_{2} \mathrm{NEt}(291 \square \mathrm{L}, 1.67 \mathrm{mmol})$ in $\mathrm{CH}_{2} \mathrm{Cl}_{2}(1.1 \mathrm{~mL})$ was added $\mathrm{BOMCl}(139 \mathrm{LL}, 1.00 \mathrm{mmol})$ at room temperature. After stirring for $38 \mathrm{~h}$, the reaction was quenched with saturated $\mathrm{NH}_{4} \mathrm{Cl}$ aq. $(6 \mathrm{~mL})$ and extracted with $\mathrm{CHCl}_{3}(2 \times 10 \mathrm{~mL})$. The combined organic layers were dried over $\mathrm{Na}_{2} \mathrm{SO}_{4}$, filtered, and concentrated. Preparative TLC (hexanes/EtOAc $=4 / 1)$ provided BOM ether $24(138 \mathrm{mg}, 100 \%)$ as a colorless oil: ${ }^{1} \mathrm{H}-\mathrm{NMR}(500 \mathrm{MHz}$, $\left.\mathrm{CDCl}_{3}\right) \square 7.33(\mathrm{~m}, 5 \mathrm{H}), 7.23(\mathrm{~d}, J=8.6 \mathrm{~Hz}, 2 \mathrm{H}), 6.85(\mathrm{~d}, J=8.6 \mathrm{~Hz}, 2 \mathrm{H}), 4.99$ (ddd, $J=11.0,1.3,1.3 \mathrm{~Hz}$, $1 \mathrm{H}), 4.79(\mathrm{~d}, J=6.7 \mathrm{~Hz}, 1 \mathrm{H}), 4.74(\mathrm{~d}, J=6.7 \mathrm{~Hz}, 1 \mathrm{H}), 4.64(\mathrm{~d}, J=11.6 \mathrm{~Hz}, 1 \mathrm{H}), 4.61(\mathrm{~d}, J=11.6 \mathrm{~Hz}, 1 \mathrm{H})$, $4.39(\mathrm{~s}, 2 \mathrm{H}), 3.79(\mathrm{~s}, 3 \mathrm{H}), 3.56(\mathrm{dd}, J=9.1,4.4 \mathrm{~Hz}, 1 \mathrm{H}), 3.32(\mathrm{dd}, J=9.1,3.3 \mathrm{~Hz}, 1 \mathrm{H}), 3.31(\mathrm{dd}, J=7.8$, $6.9 \mathrm{~Hz}, 1 \mathrm{H}), 2.62(\mathrm{~m}, 1 \mathrm{H}), 2.05(\mathrm{~m}, 1 \mathrm{H}), 1.64(\mathrm{~d}, J=1.3 \mathrm{~Hz}, 3 \mathrm{H}), 1.58(\mathrm{~d}, J=1.3 \mathrm{~Hz}, 3 \mathrm{H}), 1.03(\mathrm{~d}, J=6.9$ $\mathrm{Hz}, 3 \mathrm{H}), 0.97$ (d, $J=6.7 \mathrm{~Hz}, 3 \mathrm{H})$.

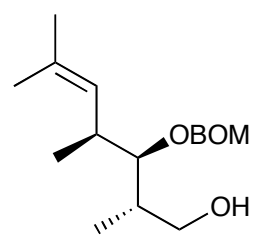

25

Alcohol 25. At $0{ }^{\circ} \mathrm{C}$, a solution of $24(140 \mathrm{mg}, 0.340 \mathrm{mmol})$ in $\mathrm{CH}_{2} \mathrm{Cl}_{2}(2.75 \mathrm{~mL})$ was treated with $\mathrm{H}_{2} \mathrm{O}$ $(0.14 \mathrm{~mL})$ and $\mathrm{DDQ}(84.9 \mathrm{mg}, 0.374 \mathrm{mmol})$, and stirred for $1 \mathrm{~h} 20 \mathrm{~min}$. The reaction was quenched with saturated $\mathrm{NaHCO}_{3}$ aq. ( $4 \mathrm{~mL}$ ) and the layers separated. The aqueous layer was then extracted with $\mathrm{CH}_{2} \mathrm{Cl}_{2}(2 \times 10 \mathrm{~mL})$. The combined organic layers were dried over $\mathrm{Na}_{2} \mathrm{SO}_{4}$, filtered, and concentrated. Flash chromatography (hexanes/EtOAc $=20 / 1 \square 1 / 1)$ provided primary alcohol $25(85.4 \mathrm{mg}, 86 \%)$ as a colorless oil: ${ }^{1} \mathrm{H}-\mathrm{NMR}\left(500 \mathrm{MHz}, \mathrm{CDCl}_{3}\right) \square 7.34(\mathrm{~m}, 5 \mathrm{H}), 4.98(\mathrm{ddd}, J=9.5,1.1,0.9 \mathrm{~Hz}, 1 \mathrm{H}), 4.82(\mathrm{~d}, J=$ 
$6.6 \mathrm{~Hz}, 1 \mathrm{H}), 4.77(\mathrm{~d}, J=6.6 \mathrm{~Hz}, 1 \mathrm{H}), 4.73(\mathrm{~d}, J=11.9 \mathrm{~Hz}, 1 \mathrm{H}), 4.60(\mathrm{~d}, J=11.9 \mathrm{~Hz}, 1 \mathrm{H}), 3.85(\mathrm{dd}, J=$ 11.2, $3.7 \mathrm{~Hz}, 1 \mathrm{H}), 3.54(\mathrm{dd}, J=11.2,4.4 \mathrm{~Hz}, 1 \mathrm{H}), 3.37(\mathrm{dd}, J=6.3,5.6 \mathrm{~Hz}, 1 \mathrm{H}), 2.65(\mathrm{~m}, 1 \mathrm{H}), 1.85(\mathrm{~m}$, $1 \mathrm{H}), 1.67(\mathrm{~d}, J=0.9 \mathrm{~Hz}, 3 \mathrm{H}), 1.62(\mathrm{~d}, J=1.1 \mathrm{~Hz}, 3 \mathrm{H}), 1.60(\mathrm{br} \mathrm{s}, 1 \mathrm{H}), 1.05(\mathrm{~d}, J=7.1 \mathrm{~Hz}, 3 \mathrm{H}), 0.98(\mathrm{~d}, J$ $=6.8 \mathrm{~Hz}, 3 \mathrm{H})$.

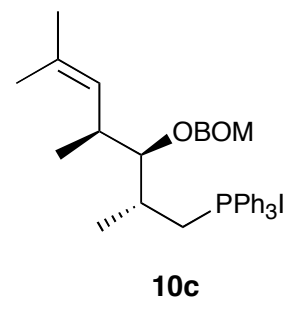

Wittig salt 10c. A solution of $25(83.2 \mathrm{mg}, 0.275 \mathrm{mmol}), \mathrm{PPh}_{3}(108.4 \mathrm{mg}, 0.413 \mathrm{mmol})$ and imidazole (28.1 $\mathrm{mg}, 0.413 \mathrm{mmol})$ in benzene $\cdot \mathrm{Et}_{2} \mathrm{O}(1: 2)(2.75 \mathrm{~mL})$ was cooled to $0{ }^{\circ} \mathrm{C}$, and treated with iodine (104.9 $\mathrm{mg}, 0.413 \mathrm{mmol})$. The reaction mixture was warmed to room temperature and stirred for $3.5 \mathrm{~h}$. The reaction was then quenched with saturated $\mathrm{NaHCO}_{3}$ aq. $(5 \mathrm{~mL})$ and the layers separated. The aqueous layer was extracted with $\mathrm{Et}_{2} \mathrm{O}(3 \times 10 \mathrm{~mL})$, and the combined organics were dried over $\mathrm{Na}_{2} \mathrm{SO}_{4}$, filtered, and concentrated. Flash chromatography (hexanes/EtOAc $=30 / 1$ ) provided a mixture of the desired alkyl iodide 9c and $\mathrm{PPh}_{3}$, which was taken onto the next step without further purification.

To the neat alkyl iodide 9c (mixed with $\mathrm{PPh}_{3}$ ) was added additional $\mathrm{PPh}_{3}(722.6 \mathrm{mg}, 2.75 \mathrm{mmol}$ ) and $i$ $\operatorname{Pr}_{2} \mathrm{NEt}(0.39 \mathrm{~mL})$ at room temperature. The mixture was warmed to $100{ }^{\circ} \mathrm{C}$ and stirred for $17 \mathrm{~h}$. The reaction mixture was cooled to room temperature and diluted with $\mathrm{CH}_{2} \mathrm{Cl}_{2}(0.5 \mathrm{~mL})$. The resulting solution was loaded onto flash chromatography $\left(\mathrm{CH}_{2} \mathrm{Cl}_{2} / \mathrm{CH}_{3} \mathrm{CN}=5 / 1 \square\right.$ 2/1) to give Wittig salt 10c (129.5 mg, $69 \%$ for 2 steps) and the mixture of cyclopentane byproducts (24 mg, 30\% for 2 steps). Wittig salt (10c); light yellow oil; ${ }^{1} \mathrm{H}-\mathrm{NMR}\left(500 \mathrm{MHz}, \mathrm{C}_{6} \mathrm{D}_{6}\right) \square 7.85(\mathrm{~m}, 6 \mathrm{H}), 7.41(\mathrm{~m}, 2 \mathrm{H}), 7.10(\mathrm{~m}, 12 \mathrm{H}), 5.40(\mathrm{~d}, J=8.5 \mathrm{~Hz}$, $1 \mathrm{H}), 5.02(\mathrm{~d}, J=6.1 \mathrm{~Hz}, 1 \mathrm{H}), 4.95(\mathrm{~d}, J=6.1 \mathrm{~Hz}, 1 \mathrm{H}), 4.87(\mathrm{~m}, 1 \mathrm{H}), 4.74(\mathrm{~d}, J=12.2 \mathrm{~Hz}, 1 \mathrm{H}), 4.65(\mathrm{~d}, J=$ $12.2 \mathrm{~Hz}, 1 \mathrm{H}$ ), 4.34 (apparent $\mathrm{t}, J=14.9 \mathrm{~Hz}, 1 \mathrm{H}), 4.15$ (dd, $J=6.0,5.2 \mathrm{~Hz}, 1 \mathrm{H}), 2.75(\mathrm{~m}, 1 \mathrm{H}), 2.15(\mathrm{~m}$, $1 \mathrm{H}), 1.64(\mathrm{~s}, 3 \mathrm{H}), 1.60(\mathrm{~s}, 3 \mathrm{H}), 1.02(\mathrm{~d}, J=6.7 \mathrm{~Hz}, 3 \mathrm{H}), 0.95(\mathrm{~d}, J=6.8 \mathrm{~Hz}, 3 \mathrm{H})$; high resolution mass spectrum $\left(\mathrm{ES}^{+}\right) \mathrm{m} / \mathrm{z} 537.2921\left[(\mathrm{M}-\mathrm{I})^{+}\right.$; calcd for $\left.\mathrm{C}_{36} \mathrm{H}_{42} \mathrm{O}_{2} \mathrm{P}: 537.2922\right]$.

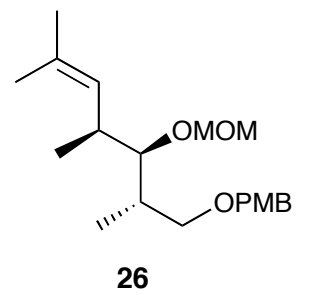

MOM ether 26. To a solution of alcohol (-)-19 (85.0 mg, $0.291 \mathrm{mmol})$ and $i$ - $\operatorname{Pr}_{2} \mathrm{NEt}(304 \mathrm{~L}, 1.75 \mathrm{mmol})$ in $\mathrm{CH}_{2} \mathrm{Cl}_{2}(0.97 \mathrm{~mL})$ was added $\mathrm{MOMCl}(88.4 \mathrm{~L}, 1.16 \mathrm{mmol})$ at room temperature. After stirring for $21 \mathrm{~h}$, 
the reaction was quenched with saturated $\mathrm{NH}_{4} \mathrm{Cl}$ aq. $(4 \mathrm{~mL})$, and extracted with $\mathrm{CHCl}_{3}(3 \times 10 \mathrm{~mL})$. The combined organic layers were dried over $\mathrm{Na}_{2} \mathrm{SO}_{4}$, filtered, and concentrated. Flash chromatography (hexanes/EtOAc $=15 / 1)$ afforded MOM ether $26(98.0 \mathrm{mg}, 100 \%)$ as a colorless oil: ${ }^{1} \mathrm{H}-\mathrm{NMR}(500 \mathrm{MHz}$, $\left.\mathrm{CDCl}_{3}\right) \square 7.25(\mathrm{~d}, J=8.5 \mathrm{~Hz}, 2 \mathrm{H}), 6.87(\mathrm{~d}, J=8.5 \mathrm{~Hz}, 2 \mathrm{H}), 4.97(\mathrm{~d}, J=9.5 \mathrm{~Hz}, 1 \mathrm{H}), 4.64(\mathrm{~d}, J=6.6 \mathrm{~Hz}$, $1 \mathrm{H}), 4.61(\mathrm{~d}, J=6.6 \mathrm{~Hz}, 1 \mathrm{H}), 4.41(\mathrm{~s}, 2 \mathrm{H}), 3.80(\mathrm{~s}, 3 \mathrm{H}), 3.53(\mathrm{dd}, J=9.1,4.4 \mathrm{~Hz}, 1 \mathrm{H}), 3.38(\mathrm{~s}, 3 \mathrm{H}), 3.29$ (apparent t, $J=8.4 \mathrm{~Hz}, 1 \mathrm{H}$ ), 3.23 (apparent t, $J=6.2 \mathrm{~Hz}, 1 \mathrm{H}), 2.59(\mathrm{~m}, 1 \mathrm{H}), 2.02(\mathrm{~m}, 1 \mathrm{H}), 1.66(\mathrm{~s}, 3 \mathrm{H})$, $1.58(\mathrm{~s}, 3 \mathrm{H}), 1.02$ (d, $J=7.0 \mathrm{~Hz}, 3 \mathrm{H}), 0.95$ (d, $J=6.7 \mathrm{~Hz}, 3 \mathrm{H}) ;{ }^{13} \mathrm{C}-\mathrm{NMR}\left(125 \mathrm{MHz}, \mathrm{CDCl}_{3}\right) \square$ 159.3, 131.0, 130.5, 129.2 (2C), 128.4, 113.7 (2C), 98.2, 85.7, 72.7, 72.3, 56.0, 55.3, 36.6, 35.1, 25.8, 17.9, 16.6, 15.1.

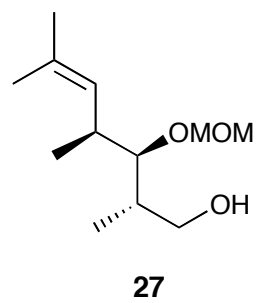

Alcohol 27. A solution of $26(92.3 \mathrm{mg}, 0.275 \mathrm{mmol})$ in $\mathrm{CH}_{2} \mathrm{Cl}_{2}(2.75 \mathrm{~mL})$ was cooled to $0{ }^{\circ} \mathrm{C}$, treated with $\mathrm{H}_{2} \mathrm{O}(0.11 \mathrm{~mL}), \mathrm{DDQ}(68.6 \mathrm{mg}, 0.302 \mathrm{mmol})$ and stirred for $1 \mathrm{~h} 20 \mathrm{~min}$. The reaction was quenched with saturated $\mathrm{NaHCO}_{3}$ aq. (4 mL) and the layers separated. The aqueous layer was then extracted with $\mathrm{CH}_{2} \mathrm{Cl}_{2}(2 \times 10 \mathrm{~mL})$. The combined organic layers were dried over $\mathrm{Na}_{2} \mathrm{SO}_{4}$, filtered, and concentrated. Flash chromatography (hexanes/EtOAc $=20 / 1 \square 1 / 1)$ provided primary alcohol $27(60.2 \mathrm{mg}, 100 \%)$ as a colorless oil: ${ }^{1} \mathrm{H}-\mathrm{NMR}\left(500 \mathrm{MHz}, \mathrm{CDCl}_{3}\right) \square 4.99(\mathrm{~d}, J=9.5 \mathrm{~Hz}, 1 \mathrm{H}), 4.68(\mathrm{~d}, J=6.5 \mathrm{~Hz}, 1 \mathrm{H}), 4.65(\mathrm{~d}, J=$ $6.5 \mathrm{~Hz}, 1 \mathrm{H}), 3.83(\mathrm{dd}, J=11.2,3.6 \mathrm{~Hz}, 1 \mathrm{H}), 3.51(\mathrm{dd}, J=11.2,4.2 \mathrm{~Hz}, 1 \mathrm{H}), 3.43(\mathrm{~s}, 3 \mathrm{H}), 3.30(\mathrm{dd}, J=$ 6.5, $5.4 \mathrm{~Hz}, 1 \mathrm{H}), 2.63(\mathrm{~m}, 1 \mathrm{H}), 1.82(\mathrm{~m}, 1 \mathrm{H}), 1.69(\mathrm{~s}, 3 \mathrm{H}), 1.62(\mathrm{~s}, 3 \mathrm{H}), 1.55(\mathrm{br} \mathrm{s}, 1 \mathrm{H}), 1.04(\mathrm{~d}, J=7.1 \mathrm{~Hz}$, $3 \mathrm{H}), 0.96(\mathrm{~d}, J=6.8 \mathrm{~Hz}, 3 \mathrm{H})$.

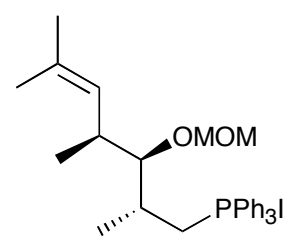

10d

Wittig salt 10d. A solution of $27(56.0 \mathrm{mg}, 0.259 \mathrm{mmol}), \mathrm{PPh}_{3}(102.0 \mathrm{mg}, 0.389 \mathrm{mmol})$ and imidazole (26.5 mg, $0.389 \mathrm{mmol})$ in benzene $\cdot \mathrm{Et}_{2} \mathrm{O}(1: 2)(2.6 \mathrm{~mL})$ was cooled to $0{ }^{\circ} \mathrm{C}$ and treated with iodine (98.7 $\mathrm{mg}, 0.389 \mathrm{mmol})$. The reaction mixture was warmed to room temperature and stirred for $3.5 \mathrm{~h}$. The reaction was then quenched with saturated aq. $\mathrm{NaHCO}_{3}(5 \mathrm{~mL})$. The mixture was extracted with $\mathrm{Et}_{2} \mathrm{O}(3 \mathrm{x}$ $10 \mathrm{~mL}$ ) and the combined organics were dried over $\mathrm{Na}_{2} \mathrm{SO}_{4}$, filtered, and concentrated. Flash 
chromatography (hexanes/EtOAc $=30 / 1$ ) provided a mixture of the desired alkyl iodide $9 \mathrm{~d}$ and $\mathrm{PPh}_{3}$, which was taken onto the next step without further purification.

To the neat alkyl iodide9d (mixed with $\mathrm{PPh}_{3}$ ) was added additional $\mathrm{PPh}_{3}(679 \mathrm{mg}, 2.59 \mathrm{mmol}$ ) and $i$ $\mathrm{Pr}_{2} \mathrm{NEt}(0.37 \mathrm{~mL})$ at room temperature. The mixture was warmed to $100{ }^{\circ} \mathrm{C}$ and stirred for $17 \mathrm{~h}$. The reaction mixture was cooled to room temperature and diluted with $\mathrm{CH}_{2} \mathrm{Cl}_{2}(0.5 \mathrm{~mL})$. The resulting solution was directly purified via flash chromatography $\left(\mathrm{CH}_{2} \mathrm{Cl}_{2} / \mathrm{CH}_{3} \mathrm{CN}=5 / 1 \square 2 / 1\right)$ to give the Wittig salt 10d (104.5 mg, 69\% for 2 steps) and the mixture of cyclopentane byproducts ( $12.2 \mathrm{mg}, 24 \%$ for 2 steps).

Wittig salt (10d); light yellow oil; ${ }^{1} \mathrm{H}-\mathrm{NMR}\left(500 \mathrm{MHz}, \mathrm{C}_{6} \mathrm{D}_{6}\right) \square 7.86(\mathrm{~m}, 6 \mathrm{H}), 7.15(\mathrm{~m}, 9 \mathrm{H}), 5.39(\mathrm{~d}, J=9.3$ $\mathrm{Hz}, 1 \mathrm{H}), 5.08(\mathrm{~m}, 1 \mathrm{H}), 4.88(\mathrm{~d}, J=5.9 \mathrm{~Hz}, 1 \mathrm{H}), 4.79(\mathrm{~d}, J=5.9 \mathrm{~Hz}, 1 \mathrm{H}), 4.32$ (apparent t, $J=15.0 \mathrm{~Hz}$, $1 \mathrm{H}), 4.10$ (apparent t, $J=5.4 \mathrm{~Hz}, 1 \mathrm{H}), 3.28(\mathrm{~s}, 3 \mathrm{H}), 2.73(\mathrm{~m}, 1 \mathrm{H}), 2.11(\mathrm{~m}, 1 \mathrm{H}), 1.66(\mathrm{~s}, 3 \mathrm{H}), 1.60(\mathrm{~s}, 3 \mathrm{H})$, $1.01(\mathrm{~d}, J=6.7 \mathrm{~Hz}, 3 \mathrm{H}), 0.96(\mathrm{~d}, J=6.8 \mathrm{~Hz}, 3 \mathrm{H})$; high resolution mass spectrum $\left(\mathrm{ES}^{+}\right) \mathrm{m} / \mathrm{z} 461.2607$ $\left[(\mathrm{M}-\mathrm{I})^{+}\right.$; calcd for $\left.\mathrm{C}_{30} \mathrm{H}_{38} \mathrm{O}_{2} \mathrm{P}: 461.2609\right]$.

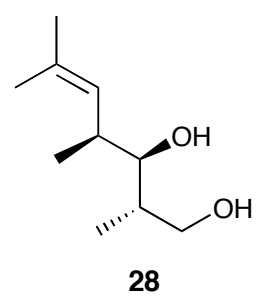

Diol 28. At $0{ }^{\circ} \mathrm{C}$, a solution of (-)-19 (96.5 mg, $\left.0.330 \mathrm{mmol}\right)$ in $\mathrm{CH}_{3} \mathrm{CN} \cdot \mathrm{H}_{2} \mathrm{O}(20: 1)(3.3 \mathrm{~mL})$ was treated with DDQ (82.5 mg, $0.364 \mathrm{mmol}$ ), stirred for $10 \mathrm{~min}$., warmed to room temperature, and then stirred for 2 h. The reaction was then quenched with saturated $\mathrm{NaHCO}_{3}$ aq. $(10 \mathrm{~mL})$ and the layers separated. The aqueous layer was then extracted with $\mathrm{Et}_{2} \mathrm{O}(2 \times 10 \mathrm{~mL})$. The combined organic layers were dried over $\mathrm{Na}_{2} \mathrm{SO}_{4}$, filtered, and concentrated. Flash chromatography (hexanes/EtOAc $=10 / 1 \square 1 / 1$ ) provided the diol 28 (38.4 mg, 68\%) as a colorless oil: ${ }^{1} \mathrm{H}-\mathrm{NMR}\left(500 \mathrm{MHz}, \mathrm{CDCl}_{3}\right) \square 5.03$ (ddd, $J=9.3,1.4,1.4 \mathrm{~Hz}$, $1 \mathrm{H}$ ), 3.77 (dd, $J=10.8,3.5 \mathrm{~Hz}, 1 \mathrm{H}$ ), $3.62(\mathrm{dd}, J=10.8,6.5 \mathrm{~Hz}, 1 \mathrm{H}$ ), $3.40(\mathrm{dd}, J=6.7,5.4 \mathrm{~Hz}, 1 \mathrm{H}$ ), 2.59 (m, 1H), 2.07 (br s, 2H), $1.82(\mathrm{~m}, 1 \mathrm{H}), 1.71(\mathrm{~d}, J=1.4 \mathrm{~Hz}, 3 \mathrm{H}), 1.64(\mathrm{~d}, J=1.4 \mathrm{~Hz}, 3 \mathrm{H}), 0.99(\mathrm{~d}, J=6.8$ $\mathrm{Hz}, 3 \mathrm{H}), 0.97(\mathrm{~d}, J=7.1 \mathrm{~Hz}, 3 \mathrm{H})$.

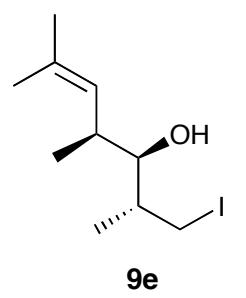

lodide 9e. A solution of $28(38.2 \mathrm{mg}, 0.222 \mathrm{mmol}), \mathrm{PPh}_{3}(87.4 \mathrm{mg}, 0.333 \mathrm{mmol})$ and imidazole $(22.7 \mathrm{mg}$, $0.333)$ in benzene $\mathrm{Et}_{2} \mathrm{O}(1: 2)(2.2 \mathrm{~mL})$ was cooled to $0{ }^{\circ} \mathrm{C}$, and treated with iodine (84.6 $\left.\mathrm{mg}, 0.333 \mathrm{mmol}\right)$. The mixture was warmed to room temperature and stirred for $25 \mathrm{~min}$. The reaction was then quenched 
with saturated $\mathrm{NaHCO}_{3}$ aq. $(3 \mathrm{~mL})$. The layers were separated and the aqueous layer was extracted with $\mathrm{Et}_{2} \mathrm{O}(2 \times 10 \mathrm{~mL})$. The combined organic extracts were dried over $\mathrm{Na}_{2} \mathrm{SO}_{4}$, filtered, and concentrated . Flash chromatography (hexanes/EtOAc $=40 / 1 \square 20 / 1)$ provided alkyl iodide 9 e $(21.9 \mathrm{mg}, 33 \%)$ as a colorless oil: ${ }^{1} \mathrm{H}-\mathrm{NMR}\left(500 \mathrm{MHz}, \mathrm{CDCl}_{3}\right) \square 5.04(\mathrm{ddd}, J=9.2,1.3,1.3 \mathrm{~Hz}, 1 \mathrm{H}), 3.41(\mathrm{dd}, J=9.6,3.0 \mathrm{~Hz}$, $1 \mathrm{H}$ ), $3.33(\mathrm{dd}, J=9.6,6.9 \mathrm{~Hz}, 1 \mathrm{H}), 3.27(\mathrm{dd}, J=7.0,4.9 \mathrm{~Hz}, 1 \mathrm{H}), 2.57(\mathrm{~m}, 1 \mathrm{H}), 1.72(\mathrm{~d}, J=1.3 \mathrm{~Hz}, 3 \mathrm{H})$, $1.65(\mathrm{~d}, J=1.3 \mathrm{~Hz}, 3 \mathrm{H}), 1.57(\mathrm{~m}, 1 \mathrm{H}), 1.03(\mathrm{~d}, J=6.7 \mathrm{~Hz}, 3 \mathrm{H}), 0.96(\mathrm{~d}, J=6.8 \mathrm{~Hz}, 3 \mathrm{H})$.

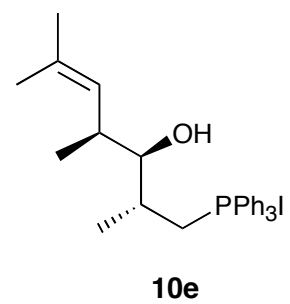

Wittig salt 10e. To the neat alkyl iodide $9 \mathrm{e}(21.4 \mathrm{mg}, 0.0718 \mathrm{mmol})$ was added $\mathrm{PPh}_{3}(188.4 \mathrm{mg}, 0.718$ $\mathrm{mmol})$ and $i-\mathrm{Pr}_{2} \mathrm{NEt}(0.10 \mathrm{~mL})$ at room temperature. The resulting solution was warmed to $100{ }^{\circ} \mathrm{C}$ and after $16 \mathrm{~h}$, the reaction mixture was cooled to room temperature and diluted with $\mathrm{CH}_{2} \mathrm{Cl}_{2}(0.5 \mathrm{~mL})$. The solution was directly purified via flash chromatography $\left(\mathrm{CH}_{2} \mathrm{Cl}_{2} / \mathrm{CH}_{3} \mathrm{CN}=5 / 1 \square 3 / 1\right)$ to provide the Wittig salt $10 \mathrm{e}(24.8 \mathrm{mg}, 62 \%)$ as a light yellow solid and the mixture of cyclized byproducts $(4.6 \mathrm{mg}, 38 \%)$ : Wittig salt (10e): ${ }^{1} \mathrm{H}-\mathrm{NMR}\left(500 \mathrm{MHz}, \mathrm{CD}_{3} \mathrm{OD}\right) \mathrm{7}$ 7.89-7.72 (m, $\left.15 \mathrm{H}\right), 4.90(\mathrm{~d}, J=8.5 \mathrm{~Hz}, 1 \mathrm{H}), 3.67(\mathrm{~m}$, $3 \mathrm{H}), 2.52(\mathrm{~m}, 1 \mathrm{H}), 2.08(\mathrm{~m}, 1 \mathrm{H}), 1.53(\mathrm{~s}, 3 \mathrm{H}), 1.46(\mathrm{~s}, 3 \mathrm{H}), 0.88(\mathrm{~d}, J=6.7 \mathrm{~Hz}, 3 \mathrm{H}), 0.82(\mathrm{~d}, J=6.8 \mathrm{~Hz}$, $3 \mathrm{H}$ ); high resolution mass spectrum $\left(\mathrm{ES}^{+}\right) \mathrm{m} / \mathrm{z} 417.2362$ [(M-I)+; calcd for $\mathrm{C}_{28} \mathrm{H}_{34} \mathrm{OP}: 417.2347$ ].

\section{(+)-Discodermolide System (Beginning from (-)-Common Precursor):}

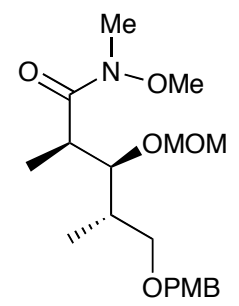

$(-)-29$

MOM Ether (-)-29. A solution of common precursor (-)-CP $(7.0 \mathrm{~g}, 21.6 \mathrm{mmol})$ and $i-\operatorname{Pr}_{2} \mathrm{NEt}(7.5 \mathrm{~mL}$, $43.2 \mathrm{mmol})$ in $\mathrm{CH}_{2} \mathrm{Cl}_{2}(210 \mathrm{~mL})$ was cooled to $0{ }^{\circ} \mathrm{C}$ and $\mathrm{MOMCl}(2.42 \mathrm{~mL}, 32.0 \mathrm{mmol})$ was added. The mixture was stirred for $2 \mathrm{~h}$, at which time another portion of $\mathrm{MOMCl}$ was added (this was repeated until no $\mathrm{SM}$ remained). The reaction was quenched by addition of a saturated aqueous solution of $\mathrm{NH}_{4} \mathrm{Cl}(50 \mathrm{~mL})$. The aqueous layer was extracted $\left(3 \times 100 \mathrm{~mL}\right.$ of $\left.\mathrm{Et}_{2} \mathrm{O}\right)$, and the combined organic layers were washed with water, saturated $\mathrm{NaHCO}_{3}(2 \times 50 \mathrm{~mL})$, and brine. The organic layer was dried over $\mathrm{MgSO}_{4}$, filtered, and concentrated to yield (-)-29 (7.5 g, 95\%) as a clear, colorless oil. The product was purified via flash 
chromatography (15\% ethyl acetate/hexanes): $[\square]_{D}^{23}-20.8^{\circ}\left(c 4.8, \mathrm{CHCl}_{3}\right)$; IR ( $\left.\mathrm{NaCl}\right) 2937,1662,1612$, 1513, 1464, 1379, 1248, 1090, $1034 \mathrm{~cm}^{-1}$; ${ }^{1} \mathrm{H}-\mathrm{NMR}\left(500 \mathrm{MHz}, \mathrm{CDCl}_{3}\right) \square 7.23(\mathrm{~d}, J=8.7 \mathrm{~Hz}, 2 \mathrm{H}), 6.84(\mathrm{~d}$, $J=8.6 \mathrm{~Hz}, 2 \mathrm{H}), 4.61\left(\mathrm{ABq}, J_{\mathrm{AB}}=6.54 \mathrm{~Hz}, \mathrm{C}_{\mathrm{AB}}=17.3 \mathrm{~Hz}, 2 \mathrm{H}\right), 4.39(\mathrm{~s}, 2 \mathrm{H}), 3.78(\mathrm{~s}, 3 \mathrm{H}), 3.76(\mathrm{dd}, J=$ 6.1, $4.8 \mathrm{~Hz}, 1 \mathrm{H}), 3.58(\mathrm{~s}, 3 \mathrm{H}), 3.56(\mathrm{~d}, J=4.8 \mathrm{~Hz}, 1 \mathrm{H}), 3.34(\mathrm{~s}, 3 \mathrm{H}), 3.27(\mathrm{dd}, J=9.1,7.1 \mathrm{~Hz}, 1 \mathrm{H}), 3.13$ (m, 1H), $3.12(\mathrm{~s}, 3 \mathrm{H}), 1.97-1.91(\mathrm{~m}, 1 \mathrm{H}), 1.15(\mathrm{~d}, J=6.9 \mathrm{~Hz}, 3 \mathrm{H}), 1.03(\mathrm{~d}, J=6.9 \mathrm{~Hz}, 3 \mathrm{H}) ;{ }^{13} \mathrm{C}-\mathrm{NMR}(125$ $\left.\mathrm{MHz}, \mathrm{CDCl}_{3}\right) \square 176.8,159.0,130.7,129.1,113.4,98.3,82.3,72.7,71.8,61.0,56.1,55.1,38.4,37.1,32.2$, 15.2, 12.9; high resolution mass spectrum $\left(\mathrm{ES}^{+}\right) \mathrm{m} / \mathrm{z} 392.20554\left[(\mathrm{M}+\mathrm{Na})^{+}\right.$; calcd for $\mathrm{C}_{19} \mathrm{H}_{31} \mathrm{NO}_{6}$ : 392.204908].

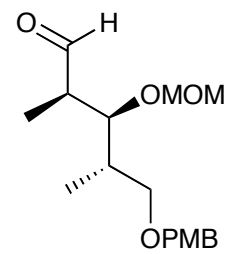

$(-)-30$

Aldehyde (-)-30. At $-10^{\circ} \mathrm{C}$, a solution of amide (-)-29 $(3.9 \mathrm{~g}, 10.5 \mathrm{mmol})$ in THF (105 mL) was treated with DIBAL-H (1.0 M in toluene, $12.7 \mathrm{~mL}$ ) via drop-wise addition down the side of the flask (10 min. addition time). The reaction was stirred for an additional $3 \mathrm{~h}$ and quenched with $\mathrm{MeOH}(1 \mathrm{~mL})$. The -10 ${ }^{\circ} \mathrm{C}$ reaction mixture was poured into saturated aqueous Rochelle's salt (100 mL), diluted with $\mathrm{Et}_{2} \mathrm{O}(150$ $\mathrm{mL}$ ), and stirred at room temperature for $30 \mathrm{~min}$. The mixture was then poured into a separatory funnel and vigorously shaken (emulsion). The layers were separated, and the organic layer was washed with saturated aqueous Rochelle's salt, water, saturated $\mathrm{NaHCO}_{3}$, and brine $(1 \times 100 \mathrm{~mL}$ each). The organic layer was dried over $\mathrm{MgSO}_{4}$, filtered, and concentrated to give (-)-30 (3.2 g, 99\%) as a clear, colorless oil, which was taken onto the next step without further purification. An analytical sample was obtained via flash chromatography (15\% EtOAc/hexanes): $[\square]_{\mathrm{D}}^{23}-30.1^{\circ}$ (c 6.5, $\left.\mathrm{CHCl}_{3}\right) ; \mathrm{IR}\left(\mathrm{CHCl}_{3}\right) 2938,2901,1513$, 1248, $1726 \mathrm{~cm}^{-1} ;{ }^{1} \mathrm{H}-\mathrm{NMR}\left(500 \mathrm{MHz}, \mathrm{CDCl}_{3}\right) \square 9.70$ (s, $\left.1 \mathrm{H}\right), 7.24$ (d, $\left.J=8.6 \mathrm{~Hz}, 2 \mathrm{H}\right), 6.87$ (d, $J=8.6 \mathrm{~Hz}$, 2H), $4.60(\mathrm{~d}, J=6.8 \mathrm{~Hz}, 1 \mathrm{H}), 4.49(\mathrm{~d}, J=6.8 \mathrm{~Hz}, 1 \mathrm{H}), 4.41(\mathrm{~s}, 2 \mathrm{H}), 4.06(\mathrm{dd}, J=8.5,3.0 \mathrm{~Hz}, 1 \mathrm{H}), 3.80(\mathrm{~s}$, 3H), 3.49 (dd, $J=8.9,5.0 \mathrm{~Hz}, 1 \mathrm{H}$ ), 3.44 (dd, $J=8.9,4.1 \mathrm{~Hz}, 1 \mathrm{H}$ ), 3.23 (s, 3H), 2.50 (ddd, $J=14.0,6.8$, $2.6 \mathrm{~Hz}, 1 \mathrm{H}), 2.03-1.95(\mathrm{~m}, 1 \mathrm{H}), 1.11(\mathrm{~d}, J=7.0 \mathrm{~Hz}, 3 \mathrm{H}), 1.00(\mathrm{~d}, J=7.0 \mathrm{~Hz}, 3 \mathrm{H}) ;{ }^{13} \mathrm{C}-\mathrm{NMR}(125 \mathrm{MHz}$, $\left.\mathrm{CDCl}_{3}\right) \square 203.9,159.1,130.4,129.1,113.7,97.7,78.9,72.7,71.4,55.6,55.2$, 48.6, 36.6, 14.5, 6.9; high resolution mass spectrum (FAB, NBA) $m / z 333.1665$ [(M+Na) ${ }^{+}$; calcd for $\mathrm{C}_{17} \mathrm{H}_{26} \mathrm{O}_{5} \mathrm{Na}$ : 333.16775$]$. 


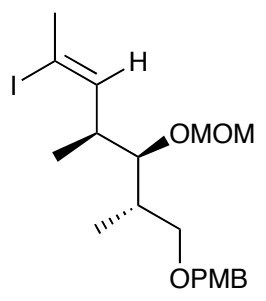

$(+)-B$

(+) - Fragment B. At $0{ }^{\circ} \mathrm{C}$, a suspension of $\mathrm{EtPh}_{3} \mathrm{PI}\left(1.34 \mathrm{~g}, 3.06 \mathrm{mmol}\right.$, dried at $70{ }^{\circ} \mathrm{C} / 0.2$ Torr for $\left.2 \mathrm{~h}\right)$ in THF (11 mL, dried over $4 \AA$ MS, sparged with argon) was treated with $n$-BuLi (2.4 M in hexanes, 1.27 $\mathrm{mL}, 3.06 \mathrm{mmol}$ ) over $30 \mathrm{~min}$. to form a dark red solution. After an additional $10 \mathrm{~min}$., the red ylide was added over $10 \mathrm{~min}$. via cannula to a cooled $\left(-78^{\circ} \mathrm{C}\right)$ solution of $\mathrm{I}_{2}(774 \mathrm{mg}, 3.06 \mathrm{mmol})$ in THF $(27 \mathrm{~mL}$ solution, prepared by adding $\mathrm{I}_{2}$ to degassed THF at $\mathrm{rt}$ and vigorously stirring for $40 \mathrm{~min}$. before cooling) such that the internal temperature did not exceed $-70{ }^{\circ} \mathrm{C}$. The resultant yellow slurry was stirred at -75 ${ }^{\circ} \mathrm{C}$ (internal) for $5 \mathrm{~min}$. and warmed to $-23^{\circ} \mathrm{C}$ (internal). NaHMDS (1.0 M in THF, $2.75 \mathrm{~mL}$ ) was added via cannula over $30 \mathrm{~min}$., and the resulting orange suspension was stirred $15 \mathrm{~min}$. further and cooled to -33 ${ }^{\circ} \mathrm{C}$ (internal). A solution of the crude aldehyde (-)-30 (475 mg, $\left.1.53 \mathrm{mmol}\right)$ in THF (5 mL) was introduced via cannula over $15 \mathrm{~min}$., and the reaction mixture was stirred at $-30^{\circ} \mathrm{C}$ for an additional $45 \mathrm{~min}$., warmed to $7{ }^{\circ} \mathrm{C}$ over $1 \mathrm{~h}$, and quenched with $\mathrm{MeOH}(2 \mathrm{~mL})$. The mixture was then suction filtered through a 6 to 8 " silica plug ( $100 \% \mathrm{Et}_{2} \mathrm{O}, 20 \mathrm{~mL}$ sintered glass frit), the filtrate was washed with saturated aqueous $\mathrm{Na}_{2} \mathrm{~S}_{2} \mathrm{O}_{3}$ and brine, dried over $\mathrm{MgSO}_{4}$, filtered, and concentrated. Flash chromatography $\left(15 \% \mathrm{CH}_{2} \mathrm{Cl}_{2} /\right.$ hexanes; then gradient elution $2 \%$ ethyl acetate/hexanes $\square 5 \%$ ethyl acetate/hexanes) furnished (+)-B (190 mg, $40 \%$ yield for two steps, 9:1 Z/E) as a clear, colorless oil. An analytical sample of the $Z$ isomer was obtained by flash chromatography (2\% ethyl acetate/hexanes): $[\square]_{\mathrm{D}}^{23}+9.2^{\circ}\left(c\right.$ 2.5, $\left.\mathrm{CHCl}_{3}\right)$; IR 2965, 2929, 2849, 1612, 1512, 1456, 1247, 1090, $1034 \mathrm{~cm}^{-1} ;{ }^{1} \mathrm{H}-\mathrm{NMR}\left(500 \mathrm{MHz}, \mathrm{CDCl}_{3}\right) \square 7.25$ (d, J=8.6 Hz, 2H), $6.87(\mathrm{~d}, J=8.6 \mathrm{~Hz}, 2 \mathrm{H}), 5.35(\mathrm{dd}, J=8.9,1.5 \mathrm{~Hz}, 1 \mathrm{H}), 4.61(\mathrm{~d}, J=6.6 \mathrm{~Hz}, 1 \mathrm{H}), 4.58(\mathrm{~d}, J=6.6 \mathrm{~Hz}, 1 \mathrm{H})$, $4.42(\mathrm{~s}, 2 \mathrm{H}), 3.80(\mathrm{~s}, 3 \mathrm{H}), 3.52(\mathrm{dd}, J=9.1,4.3 \mathrm{~Hz}, 1 \mathrm{H}), 3.38$ (apparent t, $J=5.7 \mathrm{~Hz}, 1 \mathrm{H}), 3.37(\mathrm{~s}, 3 \mathrm{H}$ ), 3.35 (dd, $J=9.1,2.0 \mathrm{~Hz}, 1 \mathrm{H}), 2.60-2.56(\mathrm{~m}, 1 \mathrm{H}), 2.46(\mathrm{~d}, J=1.5 \mathrm{~Hz}, 3 \mathrm{H}), 1.99$ (ddd, $J=10.4,6.8,4.3 \mathrm{~Hz}$, $1 \mathrm{H}), 1.04(\mathrm{~d}, J=6.9 \mathrm{~Hz}, 3 \mathrm{H}), 0.99(\mathrm{~d}, J=6.8 \mathrm{~Hz}, 3 \mathrm{H}) ;{ }^{13} \mathrm{C}-\mathrm{NMR}\left(125 \mathrm{MHz}, \mathrm{CDCl}_{3}\right) \square$ 159.0, 138.5, 130.7, 129.1, 113.6, 99.5, 98.1, 83.7, 72.6, 72.0, 55.9, 55.2, 43.6, 36.7, 33.5, 14.8, 14.2; high resolution mass spectrum (FAB, NBA) $\mathrm{m} / 2471.099989$ [(M+Na) ${ }^{+}$; calcd for $\left.\mathrm{C}_{19} \mathrm{H}_{29} \mathrm{IO}{ }_{4} \mathrm{Na}: 471.100831\right]$.

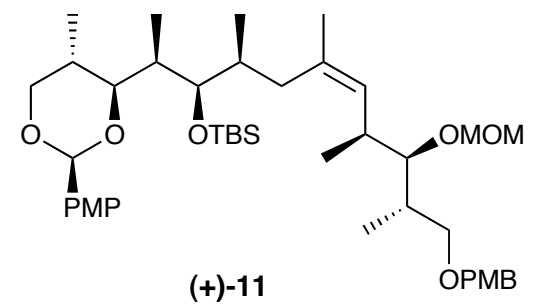


(+)-11 (Modified Negishi Coupling). A $1.0 \mathrm{M}$ solution of anhydrous $\mathrm{ZnCl}_{2}$ (11.91 mL, $1.92 \mathrm{mmol}$ ) was added via syringe to a solution of alkyl iodide (+)-A $(1.05 \mathrm{~g}, 1.9 \mathrm{mmol})$ in dry $\mathrm{Et}_{2} \mathrm{O}(8 \mathrm{~mL})$, and the resulting solution was cooled to $-78{ }^{\circ} \mathrm{C}$ and degassed by briefly pumping and backfilling with argon. $t$ BuLi (1.7 M in pentane, $13.8 \mathrm{~mL}, 5.7 \mathrm{mmol}$ ) was added via syringe over $12 \mathrm{~min}$. The resulting solution was stirred 5 min. further, evacuated $\left(1 \times 0.1\right.$ torr) and again back-filled with argon. The $-78{ }^{\circ} \mathrm{C}$ bath was then removed, and the reaction was stirred at ambient temperature for $1 \mathrm{~h}$. The resulting cloudy suspension was transferred by cannula into an intimate mixture of vinyl iodide (+)-B $(0.750 \mathrm{~g}, 1.67 \mathrm{mmol}$; 9:1 $\mathrm{Z} / E)$ and $\mathrm{Pd}\left(\mathrm{PPh}_{3}\right)_{4}(0.140 \mathrm{~g}, 0.13 \mathrm{mmol})$. The reaction mixture was stirred overnight in the absence of light, and quenched via slow addition of the reaction mixture to water $(20 \mathrm{~mL})$. The mixture was diluted with $\mathrm{Et}_{2} \mathrm{O}$, and the layers were separated. The aqueous layer was extracted $\left(3 \times \mathrm{Et}_{2} \mathrm{O}\right)$, and the combined organic layers were washed (saturated aqueous $\mathrm{NaHCO}_{3}$, brine), dried $\left(\mathrm{MgSO}_{4}\right)$, filtered and concentrated. Flash chromatography (gradient elution: 2\% EtOAc/hexanes $\square$ 5\%EtOAc/hexanes) provided (+)-11 as a light orange oil $\left(0.997 \mathrm{~g}, 80 \%\right.$ yield, $90 \%$ based on purity of vinyl iodide): $[\square]_{D}^{23}$ $+30.0^{\circ}$ ( c 0.5, $\mathrm{CHCl}_{3}$ ); IR ( $\left.\mathrm{NaCl}\right) 2958,2931,2872,2854,1615,1516,1461,1388,1249,1035 \mathrm{~cm}^{-1} ;{ }^{1} \mathrm{H}-$ $\operatorname{NMR}\left(500 \mathrm{MHz}, \mathrm{CDCl}_{3}\right) \square 7.37(\mathrm{~d}, J=8.7 \mathrm{~Hz}, 2 \mathrm{H}), 7.24(\mathrm{~d}, J=8.6 \mathrm{~Hz}, 2 \mathrm{H}), 6.86$ (apparent t, $J=8.2 \mathrm{~Hz}$, $4 \mathrm{H}), 5.39(\mathrm{~s}, 1 \mathrm{H}), 5.05(\mathrm{~d}, J=9.7 \mathrm{~Hz}, 1 \mathrm{H}), 4.61\left(\mathrm{ABq}, J_{\mathrm{AB}}=6.3 \mathrm{~Hz}, \square_{\mathrm{AB}}=14.9 \mathrm{~Hz}, 2 \mathrm{H}\right), 4.39\left(\mathrm{ABq}, J_{\mathrm{AB}}\right.$ $=11.5 \mathrm{~Hz}, \mathrm{\square \beth} \mathrm{AB}=17.1 \mathrm{~Hz}, 2 \mathrm{H}), 4.09(\mathrm{dd}, J=11.2,4.5 \mathrm{~Hz}, 1 \mathrm{H}), 3.80(\mathrm{~s}, 3 \mathrm{H}), 3.79(\mathrm{~s}, 3 \mathrm{H}), 3.63(\mathrm{dd}, J=$ 7.1, $1.9 \mathrm{~Hz}, 1 \mathrm{H}$ ), $3.51(\mathrm{~m}, 2 \mathrm{H}), 3.48$ (apparent t, $J=8.9 \mathrm{~Hz}, 1 \mathrm{H}), 3.37(\mathrm{~s}, 3 \mathrm{H}), 3.30$ (dd, $J=8.9,7.8 \mathrm{~Hz}$, 1H), 3.22 (apparent t, $J=5.9 \mathrm{~Hz}, 1 \mathrm{H}$ ), 2.61 (ddd, $J=10.1,6.7,6.7 \mathrm{~Hz}, 1 \mathrm{H}$ ), 2.31 (apparent t, $J=12.3 \mathrm{~Hz}$, 1H), 2.1-1.9 (m, 3H), 1.89 (ddd, J = 7.1, 7.1, $1.8 \mathrm{~Hz}, 1 \mathrm{H}), 1.71(\mathrm{br} \mathrm{d}, J=12.3 \mathrm{~Hz}, 1 \mathrm{H}), 1.57(\mathrm{~s}, 3 \mathrm{H}), 1.01$ (d, $J=7.1 \mathrm{~Hz}, 3 \mathrm{H}), 0.99(\mathrm{~d}, J=7.1 \mathrm{~Hz}, 3 \mathrm{H}), 0.94(\mathrm{~d}, J=6.7 \mathrm{~Hz}, 3 \mathrm{H}), 0.91(\mathrm{~s}, 9 \mathrm{H}), 0.75(\mathrm{~d}, J=7.1 \mathrm{~Hz}$, $3 \mathrm{H}), 0.74(\mathrm{~s}, 3 \mathrm{H}), 0.09$ (s, 3H), $0.42(\mathrm{~s}, 3 \mathrm{H}) ;{ }^{13} \mathrm{C}-\mathrm{NMR}\left(125 \mathrm{MHz}, \mathrm{CDCl}_{3}\right) \square$ 159.7, 159.0, 132.8, 131.5, $130.9,130.3,129.0,127.2,113.7,113.4,101.0,98.2$, 85.3, 83.3, 78.4, 73.2, 72.5, 72.3, 56.0, 55.2, 38.1, $37.6,36.7,34.5,33.7,30.7,26.1,26.2,18.4,16.3,14.8,12.6,12.1,10.8,-3.5,-3.9$; high resolution mass spectrum (ES+) $m / z 765.4767$ [(M+Na) ${ }^{+}$; calcd for $\left.\mathrm{C}_{43} \mathrm{H}_{70} \mathrm{O}_{8} \mathrm{SiNa}: 765.4738\right]$.

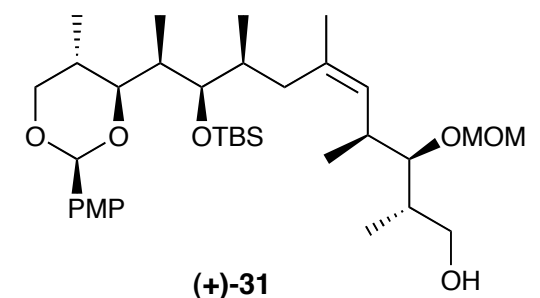

Alcohol (+)-31 (Chemoselective Hydrolysis of PMB Ether). At $0{ }^{\circ} \mathrm{C}$, a solution of PMB ether (+)-11 (850 mg, $1.14 \mathrm{mmol})$ in $\mathrm{CH}_{2} \mathrm{Cl}_{2}(10.5 \mathrm{~mL})$ was treated with $\mathrm{H}_{2} \mathrm{O}(0.5 \mathrm{~mL})$, DDQ $(312 \mathrm{mg}, 1.37 \mathrm{mmol})$ and stirred for $3 \mathrm{~h}$. The mixture was quenched with $2 \mathrm{~mL}$ of saturated $\mathrm{NaHCO}_{3}$, washed with $\mathrm{H}_{2} \mathrm{O}(2 \times 10 \mathrm{~mL})$ and the layers were separated. The aqueous layer was then extracted with $\mathrm{CH}_{2} \mathrm{Cl}_{2}(2 \times 10 \mathrm{~mL})$. The 
combined organic extracts were dried $\left(\mathrm{MgSO}_{4}\right)$, filtered, concentrated and chromatographed (10\% EtOAc/hexanes) to provided $630 \mathrm{mg}(+)-31$ (93\%); mp: $65-68{ }^{\circ} \mathrm{C}$; $[\mathrm{\square}]_{\mathrm{D}}^{23}+18.6^{\circ}\left(\mathrm{c} 6.8, \mathrm{CHCl}_{3}\right) ; \mathrm{IR}\left(\mathrm{CHCl}_{3}\right)$ 3509, 2923, 1739, 1615, 1518, 1461, 1388, 1302, $1253 \mathrm{~cm}^{-1} ;{ }^{1} \mathrm{H}-\mathrm{NMR}\left(500 \mathrm{MHz}, \mathrm{CDCl}_{3}\right) \square 7.37(\mathrm{~d}, J=$ $8.7 \mathrm{~Hz}, 2 \mathrm{H}), 6.86(\mathrm{~d}, J=8.7 \mathrm{~Hz}, 2 \mathrm{H}), 5.38(\mathrm{~s}, 1 \mathrm{H}), 5.05(\mathrm{~d}, J=10.0 \mathrm{~Hz}, 1 \mathrm{H}), 4.66(\mathrm{~d}, J=6.5 \mathrm{~Hz}, 1 \mathrm{H}), 4.64$ $(\mathrm{d}, J=6.5 \mathrm{~Hz}, 1 \mathrm{H}), 4.09$ (dd, $J=11.2,4.7 \mathrm{~Hz}, 1 \mathrm{H}), 3.84-3.80(\mathrm{~m}, 1 \mathrm{H}), 3.80(\mathrm{~s}, 3 \mathrm{H}), 3.63$ (dd, $J=7.1,1.8$ $\mathrm{Hz}, 1 \mathrm{H}), 3.51(\mathrm{dd}, J=9.9,1.7 \mathrm{~Hz}, 1 \mathrm{H}), 3.50-3.46(\mathrm{~m}, 2 \mathrm{H}), 3.42(\mathrm{~s}, 3 \mathrm{H}), 3.26(\mathrm{dd}, J=7.0,5.1 \mathrm{~Hz}, 1 \mathrm{H})$, 2.69 (dd, $J=7.6,5.2 \mathrm{~Hz}, 1 \mathrm{H}$ ), 2.64 (dd, $J=6.3,1.6 \mathrm{~Hz}, 1 \mathrm{H}$ ), 2.30 (apparent t, $J=12.2 \mathrm{~Hz}, 1 \mathrm{H}$ ), $2.09-2.03$ (m, 1H), 2.03-1.95 (m, 1H), 1.89 (ddd, $J=7.0,7.0,1.7 \mathrm{~Hz}, 1 \mathrm{H}), 1.79$ (dddd, $J=17.6,7.1,7.1,3.3 \mathrm{~Hz}$, 1H), 1.74 (br d, $J=12.7 \mathrm{~Hz}, 1 \mathrm{H}), 1.60(\mathrm{~s}, 3 \mathrm{H}), 1.02$ (d, $J=7.2 \mathrm{~Hz}, 3 \mathrm{H}), 1.01$ (d, $J=7.0 \mathrm{~Hz}, 3 \mathrm{H}), 0.94$ (d, $J$ $=6.7 \mathrm{~Hz}, 3 \mathrm{H}), 0.91(\mathrm{~s}, 9 \mathrm{H}), 0.76(\mathrm{~d}, J=6.9 \mathrm{~Hz}, 3 \mathrm{H}), 0.74(\mathrm{~d}, J=6.7 \mathrm{~Hz}, 3 \mathrm{H}), 0.05(\mathrm{~s}, 3 \mathrm{H}), 0.01(\mathrm{~s}, 3 \mathrm{H})$; ${ }^{13} \mathrm{C}-N M R\left(125 \mathrm{MHz}, \mathrm{CDCl}_{3}\right) \square$ 159.7, 133.3, 131.4, 130.0, 127.2, 113.3, 100.9, 99.0, 87.2, 83.3, 73.2, $65.0,56.2,55.1,38.1,37.6,37.3,34.6,33.8,30.7,26.1,23.2,18.3,15.2,15.0,12.5,12.0,10.7,-3.5$, -3.8; high resolution mass spectrum $\left(\mathrm{Cl}, \mathrm{NH}_{3}\right) \mathrm{m} / 2$ 645.417132 $\left[(\mathrm{M}+\mathrm{Na})^{+}\right.$; calcd for $\mathrm{C}_{35} \mathrm{H}_{62} \mathrm{O}_{7} \mathrm{SiNa}$ 645.416253.

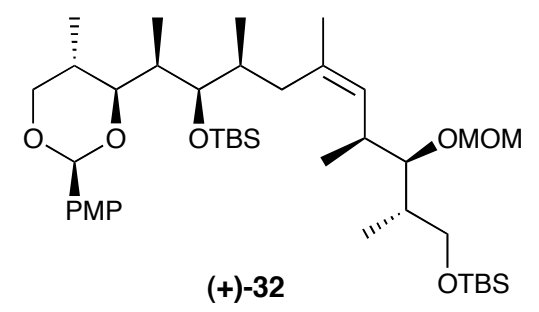

Silyl protected anisylidene acetal (+)-32. To a solution of alcohol (+)-31 (624 mg, $1.00 \mathrm{mmol})$ in $\mathrm{CH}_{2} \mathrm{Cl}_{2}$ $(10 \mathrm{~mL})$ was added imidazole (137.8 $\mathrm{mg}, 2.02 \mathrm{mmol}$ ) and TBS-Cl (227.9 mg, $1.52 \mathrm{mmol})$. The mixture was stirred for $1 \mathrm{~h}$ at ambient temperature. The reaction was then quenched with a brine solution, extracted with $\mathrm{CH}_{2} \mathrm{Cl}_{2}(3 \times 10 \mathrm{~mL})$, and washed with $\mathrm{H}_{2} \mathrm{O}(10 \mathrm{~mL})$. The organic layers were separated, dried $\left(\mathrm{MgSO}_{4}\right)$, filtered, and concentrated in vacuo. Flash chromatography $(10 \% \mathrm{EtOAc/hexanes)}$ provided (+)-32 (723 mg, 98\%); [ []$_{\mathrm{D}}^{23}+28.2^{\circ}$ (c 3.5, $\left.\mathrm{CHCl}_{3}\right)$ IR $\left(\mathrm{CHCl}_{3}\right)$ 2964, 2931, 2861, 1618, 1520, 1466, 1390, $1248 \mathrm{~cm}^{-1}$; ${ }^{1} \mathrm{H}-\mathrm{NMR}\left(500 \mathrm{MHz}, \mathrm{CDCl}_{3}\right) \square 7.37$ (d, J=8.6 Hz, 2H), $6.87(\mathrm{~d}, J=8.6 \mathrm{~Hz}, 2 \mathrm{H})$, $5.38(\mathrm{~s}, 1 \mathrm{H}), 5.10(\mathrm{~d}, J=9.9 \mathrm{~Hz}, 1 \mathrm{H}), 4.64(\mathrm{~d}, J=6.4 \mathrm{~Hz}, 1 \mathrm{H}), 4.61(\mathrm{~d}, J=6.5 \mathrm{~Hz}, 1 \mathrm{H}), 4.10$ (dd, $J=11.2$, $4.7 \mathrm{~Hz}, 1 \mathrm{H}), 3.80(\mathrm{~s}, 3 \mathrm{H}), 3.65-3.63(\mathrm{~m}, 2 \mathrm{H}), 3.54-3.44(\mathrm{~m}, 3 \mathrm{H}), 3.40(\mathrm{~s}, 3 \mathrm{H}), 3.21$ (apparent $\mathrm{t}, \mathrm{J}=5.9 \mathrm{~Hz}$, $1 \mathrm{H}$ ), 2.58 (ddd, $J=16.2,12.2,6.0 \mathrm{~Hz}, 1 \mathrm{H}$ ), 2.32 (apparent t, $J=12.2 \mathrm{~Hz}, 1 \mathrm{H}$ ) 2.10-2.04 (m, 1H), 2.04$1.94(\mathrm{~m}, 1 \mathrm{H}), 1.92-1.85(\mathrm{~m}, 1 \mathrm{H}), 1.83(\mathrm{br} d d d J=13.9,6.9,4.2 \mathrm{~Hz}, 1 \mathrm{H}), 1.75$ (apparent d, $J=12.0 \mathrm{~Hz}$, $1 \mathrm{H}), 1.60(\mathrm{~s}, 3 \mathrm{H}), 1.01(\mathrm{~d}, J=7.0 \mathrm{~Hz}, 3 \mathrm{H}), 0.94(\mathrm{~d}, J=6.6 \mathrm{~Hz}, 3 \mathrm{H}), 0.94(\mathrm{~d}, J=6.9 \mathrm{~Hz}, 3 \mathrm{H}), 0.90(\mathrm{~s}, 9 \mathrm{H})$, 0.88 (s, 9H), $0.76(\mathrm{~d}, J=6.9 \mathrm{~Hz}, 3 \mathrm{H}), 0.74(\mathrm{~d}, J=6.7 \mathrm{~Hz}, 3 \mathrm{H}), 0.04(\mathrm{~s}, 3 \mathrm{H}), 0.02$ (app s, 6H), 0.01 (s, 3H); ${ }^{13} \mathrm{C}-\mathrm{NMR}\left(125 \mathrm{MHz}, \mathrm{CHCl}_{3}\right) \square$ 159.7, 132.5, 131.4, 130.5, 127.8, 113.3, 101.0, 98.1, 84.7, 83.4, 78.3, 
73.2, 64.8, 56.0, 55.1, 38.9, 38.0, 37.4, 34.1, 33.7, 30.7, 26.1, 25.8, 23.1, 18.3, 18.2, 14.1, 12.6, 12.0, $10.7,-3.4,-3.9$; high resolution mass spectrum $\left(\mathrm{ES}^{+}\right) \mathrm{m} / \mathrm{z} 759.506365\left[(\mathrm{M}+\mathrm{Na})^{+}\right.$; calcd for $\left.\mathrm{C}_{40} \mathrm{H}_{76} \mathrm{O}_{7} \mathrm{SiNa:} 759.502732\right]$.

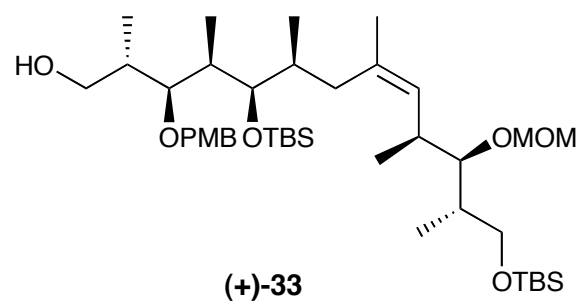

Silyl protected alcohol (+)-33. To a $-50{ }^{\circ} \mathrm{C}$ solution of silyl ether (+)-32 (724 mg, $\left.0.98 \mathrm{mmol}\right)$ in $\mathrm{CH}_{2} \mathrm{Cl}_{2}$ $(10 \mathrm{~mL})$ was added DIBAL-H (1 M in toluene, $3.9 \mathrm{~mL}, 3.9 \mathrm{mmol})$. The resulting solution was stirred for 24 $\mathrm{h}$, quenched via dropwise addition of $\mathrm{pH} 7.0$ buffer $(20 \mathrm{~mL})$, and then diluted with $\mathrm{CH}_{2} \mathrm{Cl}_{2}(20 \mathrm{~mL})$. The mixture was then added to $50 \mathrm{~mL}$ of saturated sodium potassium tartrate solution, extracted with $\mathrm{CH}_{2} \mathrm{Cl}_{2}$ $(4 \times 30 \mathrm{~mL})$, and the layers separated. The organic layer was washed with $\mathrm{H}_{2} \mathrm{O}(50 \mathrm{~mL})$, dried $\left(\mathrm{MgSO}_{4}\right)$, filtered, and concentrated. Flash chromatography (20\% EtOAc/hexanes) provided $615 \mathrm{mg}(+)-33$ (85\%) as a white foam: $[\square]_{\mathrm{D}}^{23}+3.8\left(\mathrm{c} 2.6, \mathrm{CHCl}_{3}\right)$; IR $\left(\mathrm{CHCl}_{3}\right) 3474,2957,2929,2856,1612,1514,1472,1387$, 1250, 1089, $1036 \mathrm{~cm}^{-1} ;{ }^{1} \mathrm{H}-\mathrm{NMR}\left(500 \mathrm{MHz}, \mathrm{CDCl}_{3}\right) \square 7.26$ (d, $\left.J=8.7 \mathrm{~Hz}, 2 \mathrm{H}\right), 6.87$ (d, J=8.7 Hz, 2H), $5.12(\mathrm{~d}, J=10.0 \mathrm{~Hz}, 1 \mathrm{H}), 4.65(\mathrm{~d}, J=6.4 \mathrm{~Hz}, 1 \mathrm{H}), 4.61(\mathrm{~d}, J=6.5 \mathrm{~Hz}, 1 \mathrm{H}), 4.53(\mathrm{~d}, J=10.5 \mathrm{~Hz}, 1 \mathrm{H}), 4.49$ (d, $J=10.4 \mathrm{~Hz}, 1 \mathrm{H}$ ), 3.80 (s, 3H), 3.75 (ddd, $J=11.0,5.2,3.5 \mathrm{~Hz}, 1 \mathrm{H}$ ), 3.63 (dd, $J=9.7,4.2 \mathrm{~Hz}, 1 \mathrm{H}$ ), $3.58(\mathrm{dt}, J=11.0,5.5 \mathrm{~Hz}, 1 \mathrm{H}$ ), 3.54 (dd, $J=5.1,3.6 \mathrm{~Hz}, 1 \mathrm{H}$ ), 3.48 (dd, $J=9.8,7.4 \mathrm{~Hz}, 1 \mathrm{H}$ ), 3.40 (s, 3H), 3.35 (apparent $t, J=5.5 \mathrm{~Hz}, 1 \mathrm{H}$ ), 3.21 (apparent $\mathrm{t}, J=5.8 \mathrm{~Hz}, 1 \mathrm{H}$ ), 2.73 (apparent $\mathrm{t}, J=5.6 \mathrm{~Hz}, 1 \mathrm{H}$ ), 2.57 (dq, $J=12.8,6.4 \mathrm{~Hz}, 1 \mathrm{H}$ ), 2.24 (apparent t, $J=12.3 \mathrm{~Hz}, 1 \mathrm{H}$ ), 2.00-1.88 (m, 3H), 1.86-1.80 (m, 2H), 1.64 (s, 3H), 1.05 (d, J=7.2 Hz, 3H), $1.03(\mathrm{~d}, J=7.1 \mathrm{~Hz}, 3 \mathrm{H}), 0.96-0.92(\mathrm{~m}, 6 \mathrm{H}) 0.94(\mathrm{~s}, 9 \mathrm{H}), 0.88(\mathrm{~s}, 9 \mathrm{H})$, $0.75(\mathrm{~d}, J=6.7 \mathrm{~Hz}, 3 \mathrm{H}), 0.08(\mathrm{~s}, 6 \mathrm{H}), 0.02(\mathrm{br} \mathrm{s}, 6 \mathrm{H}) ;{ }^{13} \mathrm{C}-\mathrm{NMR}\left(125 \mathrm{MHz}, \mathrm{CDCl}_{3}\right) \square$ 159.2, 132.1, 130.9, $130.2,129.3,113.8,98.1,86.3,84.7,77.3,75.2,65.2,64.8,56.0,55.2,39.7,38.4,37.3$, 36.5, 35.2, 34.2, $26.1,25.8,23.1,18.4,18.2,16.0,15.6,14.1,13.6,11.4,-3.2,-3.5,-5.4$ (2); high resolution mass spectrum $\left(\mathrm{ES}^{+}\right) \mathrm{m} / \mathrm{z} 761.516364\left[(\mathrm{M}+\mathrm{Na})^{+}\right.$; calcd for $\left.\mathrm{C}_{41} \mathrm{H}_{78} \mathrm{O}_{7} \mathrm{Si}_{2} \mathrm{Na}: 761.518382\right]$.

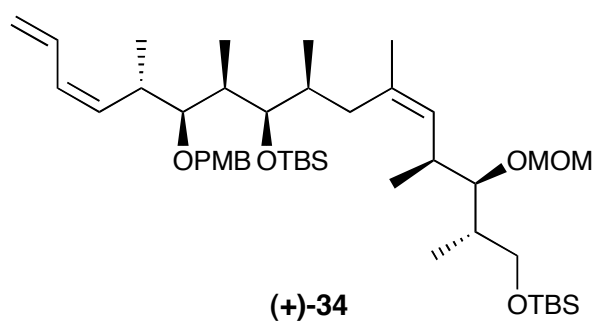


Silyl Protected Triene 34. To a $0{ }^{\circ} \mathrm{C}$ solution of alcohol (+)-33 (160 mg, $\left.0.21 \mathrm{mmol}\right)$ in $\mathrm{CH}_{2} \mathrm{Cl}_{2}(2.1 \mathrm{~mL})$ was added Dess-Martin periodinane $(87.4 \mathrm{mg}, 0.23 \mathrm{mmol})$ and $\mathrm{NaHCO}_{3}(47.4 \mathrm{mg}, 0.63 \mathrm{mmol})$. The resulting solution was stirred for $2.5 \mathrm{~h}$ and quenched with saturated $\mathrm{NaS}_{2} \mathrm{O}_{3}$ solution $(2 \mathrm{~mL})$ and saturated $\mathrm{NaHCO}_{3}$ solution $(2 \mathrm{~mL})$. The mixture was then extracted with $\mathrm{Et}_{2} \mathrm{O}(3 \times 10 \mathrm{~mL})$ and the layers were separated. The organic layer was then washed with $\mathrm{H}_{2} \mathrm{O}$, dried $\left(\mathrm{MgSO}_{4}\right)$, filtered, and concentrated. The resulting white foam 12 was used without further purification. An analytical sample was obtained via flash chromatography (3\% EtOAc / hexanes): Aldehyde 12: IR $\left(\mathrm{CHCl}_{3}\right)$ 2953, 2926, 2861, 1716, 1515, 1460, $1253 \mathrm{~cm}^{-1}$; ${ }^{1} \mathrm{H}-\mathrm{NMR}\left(500 \mathrm{MHz}, \mathrm{CDCl}_{3}\right) \square 9.80(\mathrm{~d}, J=2.5 \mathrm{~Hz}, 1 \mathrm{H}), 7.22(\mathrm{~d}, J=8.6 \mathrm{~Hz}, 2 \mathrm{H}), 6.80(\mathrm{~d}, J=8.6$ Hz, 2H), $5.13(\mathrm{~d}, J=9.8 \mathrm{~Hz}, 1 \mathrm{H}), 4.65(\mathrm{~d}, J=6.5 \mathrm{~Hz}, 1 \mathrm{H}), 4.61(\mathrm{~d}, J=6.5 \mathrm{~Hz}, 1 \mathrm{H}), 4.47(\mathrm{~s}, 2 \mathrm{H}), 3.79$ (s, $3 \mathrm{H}$ ), 3.64 (dd, $J=9.9,4.3 \mathrm{~Hz}, 1 \mathrm{H}$ ), 3.60 (dd, $J=5.3,3.2 \mathrm{~Hz}, 1 \mathrm{H}$ ), 3.57 (apparent t, $J=5.4 \mathrm{~Hz}, 1 \mathrm{H}$ ), 3.49 (dd, $J=9.3,7.1 \mathrm{~Hz}, 1 \mathrm{H}$ ), 3.41 (s, 3H), 3.22 (apparent t, $J=5.8 \mathrm{~Hz}, 1 \mathrm{H}$ ), 2.74 (ddd, $J=15.2,7.0,2.6 \mathrm{~Hz}$, $1 \mathrm{H}$ ), 2.57 (ddd, $J=16.8,13.1,6.5 \mathrm{~Hz}, 1 \mathrm{H}$ ), 2.25 (apparent t, $J=12.5 \mathrm{~Hz}, 1 \mathrm{H}$ ), $2.00-1.88(\mathrm{~m}, 2 \mathrm{H}), 1.98-$ $1.81(\mathrm{~m}, 1 \mathrm{H}), 1.78(\mathrm{bd}, J=13.1 \mathrm{~Hz}, 1 \mathrm{H}), 1.63(\mathrm{~s}, 3 \mathrm{H}), 1.12(\mathrm{~d}, J=7.0 \mathrm{~Hz}, 3 \mathrm{H}), 1.04(\mathrm{~d}, J=6.9 \mathrm{~Hz}, 3 \mathrm{H})$, 0.96-0.93 (m, 6H), $0.93(\mathrm{~s}, 9 \mathrm{H}), 0.89(\mathrm{~s}, 9 \mathrm{H}), 0.76(\mathrm{~d}, J=6.7 \mathrm{~Hz}, 3 \mathrm{H}), 0.08(\mathrm{~s}, 3 \mathrm{H}), 0.07(\mathrm{~s}, 3 \mathrm{H}), 0.03(\mathrm{~s}$, $6 \mathrm{H}) ;{ }^{13} \mathrm{C}-\mathrm{NMR}\left(125 \mathrm{MHz}, \mathrm{CDCl}_{3}\right) \square 204.4,159.2,131.9,131.0,130.2,129.0,113.7,98.1,84.7,82.6,77.0$, 76.6, 74.3, 64.8, 56.0, 55.1, 49.4, 40.1, 38.8, 36.5, 35.0, 34.2, 26.1, 25.8, 23.1, 18.4, 18.2, 16.0, 14.1, $13.4,12.1,11.3,-3.2,-3.6,-5.4$; high resolution mass spectrum $\left(\mathrm{ES}^{+}\right) \mathrm{m} / \mathrm{z} 759.506140\left[(\mathrm{M}+\mathrm{Na})^{+}\right.$; calcd for $\mathrm{C}_{41} \mathrm{H}_{76} \mathrm{O}_{7} \mathrm{Si}_{2} \mathrm{Na}$ : 759.502732].

To a $-78{ }^{\circ} \mathrm{C}$ solution of freshly distilled allyldiphenylphosphine (95 $\left.\mathrm{GL}, 0.44 \mathrm{mmol}\right)$ in THF (2.1 $\mathrm{mL}$, degassed) was added $270 \mathrm{~L}$ of $t$-butyllithium (1.7 M in pentane, $0.44 \mathrm{mmol}$ ) and the solution was stirred for $5 \mathrm{~min}$. The solution was warmed to $0{ }^{\circ} \mathrm{C}$, stirred for $30 \mathrm{~min}$. and cooled to $-78^{\circ} \mathrm{C}$. The solution was then treated with freshly distilled $\mathrm{Ti}(\mathrm{O} i-\mathrm{Pr})_{4}(131 \mathrm{GL}, 0.44 \mathrm{mmol})$ and stirred for $30 \mathrm{~min}$. A precooled $\left(-78^{\circ} \mathrm{C}\right)$ solution of aldehyde $12(160 \mathrm{mg}, 0.217 \mathrm{mmol})$ in THF $(2 \mathrm{~mL})$ was added via cannula (rinse $1 \times 2$ $\mathrm{mL})$, stirred for $1 \mathrm{~h}$, and then warmed to $0{ }^{\circ} \mathrm{C}$. lodomethane $(0.13 \mathrm{~mL}, 2.17 \mathrm{mmol})$ was added, and the solution was warmed to ambient temperature and stirred for $16 \mathrm{~h}$. The solution was quenched with $\mathrm{pH} 7.0$ buffer $(2 \mathrm{~mL})$ and extracted with $\mathrm{CH}_{2} \mathrm{Cl}_{2}(3 \times 10 \mathrm{~mL})$ and $\mathrm{Et}_{2} \mathrm{O}(3 \times 10 \mathrm{~mL})$. The combined organic layers were washed with brine, dried $\left(\mathrm{MgSO}_{4}\right)$, filtered, and concentrated. Flash chromatography (2\% EtOAc/hexanes) provided (+)-34 (102 mg, 66\% from (+)-33, 14:1 mixture of Z:E isomers) as a white foam: $[\square]_{\mathrm{D}}^{23}+30.0\left(\right.$ c 1.0, $\left.\mathrm{CHCl}_{3}\right) ; \mathrm{IR}\left(\mathrm{CHCl}_{3}\right) 3060,2940,1600,1450 \mathrm{~cm}^{-1} ;{ }^{1} \mathrm{H}-\mathrm{NMR}\left(500 \mathrm{MHz}, \mathrm{CDCl}_{3}, \mathrm{Z}\right.$ isomer) $\square 7.28(\mathrm{~d}, J=8.6 \mathrm{~Hz}, 2 \mathrm{H}), 6.87(\mathrm{~d}, J=8.6 \mathrm{~Hz}, 2 \mathrm{H}), 6.56(\mathrm{ddd}, J=16.8,10.6,10.6 \mathrm{~Hz}, 1 \mathrm{H}), 6.02$ (apparent t, $J=11.0 \mathrm{~Hz}, 1 \mathrm{H}$ ), 5.58 (apparent t, $J=10.5 \mathrm{~Hz}, 1 \mathrm{H}$ ), 5.20 (dd, $J=16.8,1.3 \mathrm{~Hz}, 1 \mathrm{H}), 5.10(\mathrm{~m}$, $2 \mathrm{H}$ ), $4.65(\mathrm{~d}, J=6.5 \mathrm{~Hz}, 1 \mathrm{H}), 4.62(\mathrm{~d}, J=6.5 \mathrm{~Hz}, 1 \mathrm{H}), 4.57$ (d, $J=10.6 \mathrm{~Hz}, 1 \mathrm{H}), 4.47(\mathrm{~d}, J=10.6 \mathrm{~Hz}, 1 \mathrm{H})$, $3.80(\mathrm{~s}, 3 \mathrm{H}), 3.64(\mathrm{dd}, J=9.6,4.3 \mathrm{~Hz}, 1 \mathrm{H}), 3.52-3.46(\mathrm{~m}, 2 \mathrm{H}), 3.41(\mathrm{~s}, 3 \mathrm{H}), 3.25(\mathrm{dd}, J=7.1,3.9 \mathrm{~Hz}, 1 \mathrm{H})$, 3.21 (apparent t, $J=5.8 \mathrm{~Hz}, 1 \mathrm{H}), 3.04-2.96(\mathrm{~m}, 1 \mathrm{H}), 2.58-2.52(\mathrm{~m}, 1 \mathrm{H}), 2.09$ (apparent t, $J=12.2 \mathrm{~Hz}, 1 \mathrm{H}$ ), 
1.88-1.79 (m, 3H), $1.75(\mathrm{br} \mathrm{d}, J=13.0 \mathrm{~Hz}, 1 \mathrm{H}), 1.60(\mathrm{~s}, 3 \mathrm{H}), 1.10(\mathrm{~d}, J=6.8 \mathrm{~Hz}, 3 \mathrm{H}), 1.02(\mathrm{~d}, J=6.8 \mathrm{~Hz}$, $3 \mathrm{H}), 0.96(\mathrm{~s}, 9 \mathrm{H}), 0.94(\mathrm{~d}, J=6.8 \mathrm{~Hz}, 3 \mathrm{H}), 0.93(\mathrm{~d}, J=6.5 \mathrm{~Hz}, 3 \mathrm{H}), 0.90(\mathrm{~s}, 9 \mathrm{H}), 0.74(\mathrm{~d}, J=6.7 \mathrm{~Hz}, 3 \mathrm{H})$, $0.11(\mathrm{~s}, 3 \mathrm{H}), 0.10(\mathrm{~s}, 3 \mathrm{H}), 0.04(\mathrm{~s}, 3 \mathrm{H}), 0.04(\mathrm{~s}, 3 \mathrm{H}) ;{ }^{13} \mathrm{C}-\mathrm{NMR}\left(125 \mathrm{MHz}, \mathrm{CDCl}_{3}, \mathrm{Z}\right.$ isomer) $\square$ 159.0, 134.5, 132.3, 132.2, 131.1, 130.6, 129.0, 128.9, 117.4, 113.6, 98.0, 84.7, 84.5, 77.0, 74.8, 64.9, 55.9, 55.1, 40.0, 38.9, 36.1, 35.4, 35.3, 34.1, 26.2, 25.9, 23.0, 18.6, 18.2, 18.1, 16.0, 14.3, 14.0, 10.7, -3.2, - 3.4, -5.4 (2); high resolution mass spectrum $\left(\mathrm{ES}^{+}\right) \mathrm{m} / 2783.5400$ [(M+Na) ${ }^{+}$; calcd for $\mathrm{C}_{44} \mathrm{H}_{80} \mathrm{O}_{6} \mathrm{Si}_{2} \mathrm{Na}$ : 783.5391].

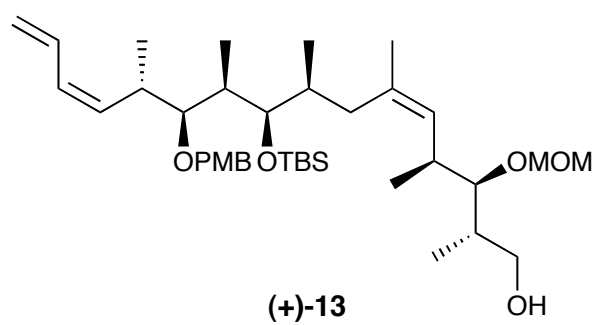

MOM ether (+)-13. Bis-TBS alcohol (+)-34 (211.1 mg, $0.278 \mathrm{mmol})$ was dissolved in a $1.0 \%$ conc. $\mathrm{HCl} / \mathrm{EtOH}$ solution $(9.3 \mathrm{~mL})(37 \% \mathrm{HCl}$ solution:EtOH $=1: 99)$ and stirred for $25 \mathrm{~min}$. at room temperature. The mixture was then neutralized with saturated $\mathrm{NaHCO}_{3}$ aq., extracted with $\mathrm{CHCl}_{3}(3 \times 50 \mathrm{~mL})$, and the combined organic layers were dried over $\mathrm{Na}_{2} \mathrm{SO}_{4}$, filtered, and concentrated. Flash chromatography (10\% 30\% EtOAc/hexanes) provided MOM-ether (+)-13 (175.6 mg, 98\%) as a colorless oil: [ $]_{\mathrm{D}}^{23}$ $+16.0^{\circ}$ ( c 2.0, $\left.\mathrm{CHCl}_{3}\right)$; IR $\left(\mathrm{CHCl}_{3}\right) 3502(\mathrm{br}), 2960$ (s), $2932(\mathrm{~s}), 2880(\mathrm{~m}), 2856(\mathrm{~m}), 1616(\mathrm{w}), 1514(\mathrm{~m})$, $1456(\mathrm{~m}), 1374(\mathrm{w}), 1302(\mathrm{w}), 1250(\mathrm{~s}), 1145(\mathrm{w}), 1091(\mathrm{~s}), 1038(\mathrm{~s}), 956(\mathrm{~m}), 905(\mathrm{w}), 835(\mathrm{~s}), 772(\mathrm{~m})$ $\mathrm{cm}^{-1}$; ${ }^{1} \mathrm{H}-\mathrm{NMR}\left(500 \mathrm{MHz}, \mathrm{CDCl}_{3}\right) \square 7.27(\mathrm{~d}, J=8.7 \mathrm{~Hz}, 2 \mathrm{H}), 6.87(\mathrm{~d}, J=8.7 \mathrm{~Hz}, 2 \mathrm{H}), 6.60$ (ddd, $J=16.7$, 10.7, $10.5 \mathrm{~Hz}, 1 \mathrm{H}$ ), 6.03 (apparent t, $J=10.9 \mathrm{~Hz}, 1 \mathrm{H}$ ), 5.58 (apparent t, $J=10.5 \mathrm{~Hz}, 1 \mathrm{H}$ ), $5.21(\mathrm{~d}, J=16.7$ $\mathrm{Hz}, 1 \mathrm{H}), 5.12(\mathrm{~d}, J=10.3 \mathrm{~Hz}, 1 \mathrm{H}), 5.02(\mathrm{~d}, J=10.1 \mathrm{~Hz}, 1 \mathrm{H}), 4.65(\mathrm{~s}, 2 \mathrm{H}), 4.57(\mathrm{~d}, J=10.6 \mathrm{~Hz}, 1 \mathrm{H}), 4.46$ (d, $J=10.6 \mathrm{~Hz}, 1 \mathrm{H}), 3.81$ (dd, $J=10.8,3.6 \mathrm{~Hz}, 1 \mathrm{H}), 3.80(\mathrm{~s}, 3 \mathrm{H}), 3.46(\mathrm{~m}, 2 \mathrm{H}), 3.43(\mathrm{~s}, 3 \mathrm{H}), 3.23(\mathrm{~m}, 2 \mathrm{H})$, $3.00(\mathrm{~m}, 1 \mathrm{H}), 2.59(\mathrm{~m}, 1 \mathrm{H}), 2.04$ (apparent t, $J=12.2 \mathrm{~Hz}, 1 \mathrm{H}), 1.79(\mathrm{~m}, 3 \mathrm{H}), 1.71$ (apparent d, $J=13.2$ Hz, 1H), 1.59 (s, 3H), 1.50 (br s, 1H), 1.10 (d, J = 6.8 Hz, 3H), 1.01 (d, J = 7.1 Hz, 6H), 0.95 (s, 9H), 0.92 $(\mathrm{d}, J=6.7 \mathrm{~Hz}, 3 \mathrm{H}), 0.72(\mathrm{~d}, J=6.7 \mathrm{~Hz}, 3 \mathrm{H}), 0.10(\mathrm{~s}, 3 \mathrm{H}), 0.09(\mathrm{~s}, 3 \mathrm{H}) ;{ }^{13} \mathrm{C}-\mathrm{NMR}\left(125 \mathrm{MHz}, \mathrm{CDCl}_{3}\right) \square$ 159.1, 134.5, 133.3, 132.3, 131.2, 130.1, 129.1, 129.0, 117.5, 113.7, 99.1, 87.6, 84.6, 77.0, 74.9, 65.0, 56.3, 55.3, 40.2, 37.4, 36.2, 35.6, 35.4, 34.8, 26.3, 23.2, 18.7, 18.6, 15.5, 15.2, 14.5, 10.7, -3.3, -3.3; high resolution mass spectrum (FAB, NBA) $\mathrm{m} / 2669.4520\left[(\mathrm{M}+\mathrm{Na})^{+}\right.$; calcd for $\mathrm{C}_{38} \mathrm{H}_{66} \mathrm{O}_{6} \mathrm{SiNa}$ : 669.4526].

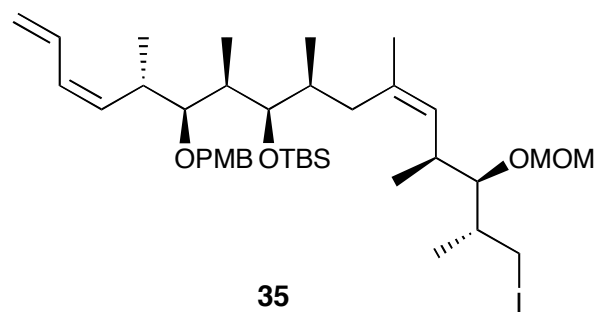


lodide 35. A solution of (+)-13 (47.9 mg, $0.0741 \mathrm{mmol}), \mathrm{PPh}_{3}(29.2 \mathrm{mg}, 0.111 \mathrm{mmol})$ and imidazole $(7.6$ $\mathrm{mg}, 0.111 \mathrm{mmol})$ in benzene $\cdot \mathrm{Et}_{2} \mathrm{O}(1: 2)(1.06 \mathrm{~mL})$ was cooled to $0{ }^{\circ} \mathrm{C}$, and treated with iodine $(28.2 \mathrm{mg}$, $0.111 \mathrm{mmol}$ ). The reaction mixture was warmed to room temperature and stirred for $12 \mathrm{~min}$. The reaction was then quenched with saturated $\mathrm{NaHCO}_{3}$ aq. $(5 \mathrm{~mL})$. The mixture was extracted with $\mathrm{Et}_{2} \mathrm{O}(3 \times 15 \mathrm{~mL})$, and the combined organics were dried over $\mathrm{Na}_{2} \mathrm{SO}_{4}$, filtered, and concentrated. Flash chromatography (15\% EtOAc/hexanes) provided a mixture of the desired alkyl iodide 35 and $\mathrm{PPh}_{3}$, which was taken onto the next step without further purification.

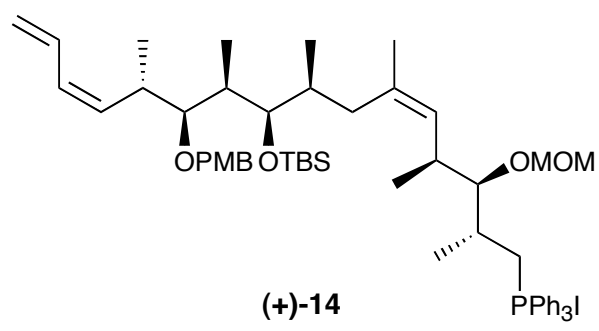

Wittig Salt (+)14. To the neat alkyl iodide 35 (mixed with $\mathrm{PPh}_{3}$ ) was added additional $\mathrm{PPh}_{3}(194 \mathrm{mg}$, $0.741 \mathrm{mmol})$ and $i-\mathrm{Pr}_{2} \mathrm{NEt}(0.37 \mathrm{~mL})$ at room temperature under argon. The mixture was warmed to 100 ${ }^{\circ} \mathrm{C}$ and stirred. After $18 \mathrm{~h}$, the reaction mixture was cooled to room temperature and diluted with $\mathrm{CH}_{2} \mathrm{Cl}_{2}$ $(0.5 \mathrm{~mL})$. The resulting solution was directly purified via flash chromatography $(20 \% \mathrm{EtOAc/hexane}$, then $15 \% \square 50 \% \mathrm{CH}_{3} \mathrm{CN} / \mathrm{CH}_{2} \mathrm{Cl}_{2}$ ) to give Wittig salt (+)-14 as a colorless oil (52.2 mg, $70 \%$ for 2 steps) and the mixture of cyclized byproducts $\left(13.1 \mathrm{mg}, 28 \%\right.$ for 2 steps): Wittig Salt $(+)-14:[\square]_{\mathrm{D}}^{23}+17.8^{\circ}(c 1.0$, $\left.\mathrm{CHCl}_{3}\right)$; IR $\left(\mathrm{CHCl}_{3}\right) 2962$ (s), 2931 (s), 2854 (m), 1612 (w), 1587 (w),1514 (m), 1456 (m), 1438 (w), 1249 (s), 1035 (s), 835 (s), 750 (s) cm ${ }^{-1}$; ${ }^{1} \mathrm{H}-\mathrm{NMR}\left(500 \mathrm{MHz}, \mathrm{CDCl}_{3}\right) \square 7.74$ (m, 15H), 7.26 (d, J = 8.6 Hz, 2H), $6.86(\mathrm{~d}, J=8.6 \mathrm{~Hz}, 2 \mathrm{H}$ ), 6.55 (ddd, $J=16.8,10.7,10.5 \mathrm{~Hz}, 1 \mathrm{H}$ ), 5.94 (apparent t, $J=11.0 \mathrm{~Hz}, 1 \mathrm{H}$ ), 5.53 (apparent t, $J=10.5 \mathrm{~Hz}, 1 \mathrm{H}), 5.13(\mathrm{~d}, J=16.8 \mathrm{~Hz}, 1 \mathrm{H}), 5.08(\mathrm{~d}, J=10.1 \mathrm{~Hz}, 1 \mathrm{H}), 5.05(\mathrm{~d}, J=10.1 \mathrm{~Hz}$, $1 \mathrm{H}), 4.70(\mathrm{~d}, J=6.1 \mathrm{~Hz}, 1 \mathrm{H}), 4.66(\mathrm{~d}, J=6.1 \mathrm{~Hz}, 1 \mathrm{H}), 4.55(\mathrm{~d}, J=10.5 \mathrm{~Hz}, 1 \mathrm{H}), 4.44(\mathrm{~d}, J=10.5 \mathrm{~Hz}, 1 \mathrm{H})$, $3.78(\mathrm{~s}, 3 \mathrm{H}), 3.72$ (apparent t, $J=16.8 \mathrm{~Hz}, 1 \mathrm{H}), 3.42$ (dd, $J=4.8,3.5 \mathrm{~Hz}, 1 \mathrm{H}), 3.34(\mathrm{~s}, 3 \mathrm{H}), 3.31$ (apparent t, $J=5.8 \mathrm{~Hz}, 1 \mathrm{H}), 3.22(\mathrm{~m}, 2 \mathrm{H}), 2.96(\mathrm{~m}, 1 \mathrm{H}), 2.50(\mathrm{~m}, 1 \mathrm{H}), 2.14(\mathrm{~m}, 1 \mathrm{H}), 1.92$ (apparent t, $J=$ $12.2 \mathrm{~Hz}, 1 \mathrm{H}), 1.73(\mathrm{~m}, 2 \mathrm{H}), 1.59(\mathrm{~m}, 1 \mathrm{H}), 1.50(\mathrm{~s}, 3 \mathrm{H}), 1.09(\mathrm{~d}, J=6.9 \mathrm{~Hz}, 3 \mathrm{H}), 0.94(\mathrm{~d}, J=6.9 \mathrm{~Hz}, 3 \mathrm{H})$, $0.90(\mathrm{~s}, 9 \mathrm{H}), 0.83$ (d, $J=6.6 \mathrm{~Hz}, 3 \mathrm{H}), 0.76(\mathrm{~d}, J=6.8 \mathrm{~Hz}, 3 \mathrm{H}), 0.65$ (d, $J=6.7 \mathrm{~Hz}, 3 \mathrm{H}), 0.08(\mathrm{~s}, 3 \mathrm{H}), 0.04$ $(\mathrm{s}, 3 \mathrm{H}) ;{ }^{13} \mathrm{C}-\mathrm{NMR}\left(125 \mathrm{MHz}, \mathrm{CDCl}_{3}\right) \square$ 159.1, 135.3, 135.3, 134.3, 133.8, 133.5, 132.1, 131.0, 130.7, 130.6, 129.1, 128.9, 118.8, 118.1, 117.6, 113.7, 99.3, 88.1, 88.0, 84.5, 75.0, 56.2, 55.3, 54.8, 39.9, 35.7, $35.5,35.3,34.2,31.7,26.2,22.9,18.7,18.5,17.9,15.6,14.8,10.7,-3.3,-3.4$; high resolution mass spectrum $\left(\mathrm{ES}^{+}\right) \mathrm{m} / \mathrm{z} 891.5537$ [(M-I)+; calcd for $\mathrm{C}_{56} \mathrm{H}_{80} \mathrm{O}_{5} \mathrm{PSi}$ : 891.5513]. Cyclized byproducts; high resolution mass spectrum $\left(\mathrm{ES}^{+}\right) \mathrm{m} / \mathrm{z} 651.4414$ [(M+Na) ${ }^{+}$; calcd for $\left.\mathrm{C}_{38} \mathrm{H}_{64} \mathrm{O}_{5} \mathrm{SiNa}: 651.4421\right]$. 


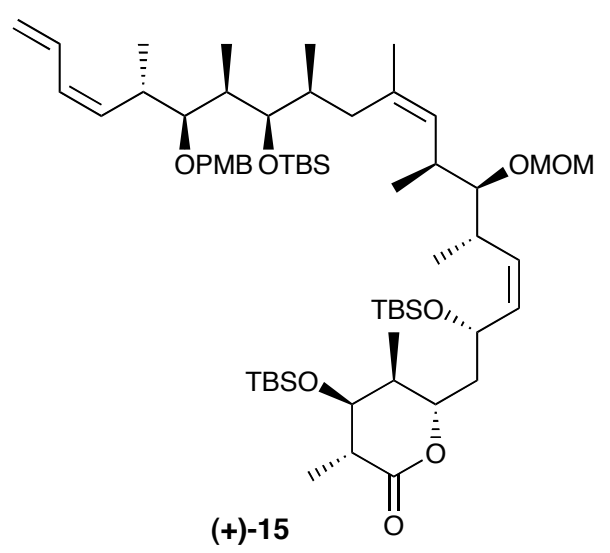

Tetraene (+)-15 (Wittig Coupling). Phosphonium salt (+)-14 (330 mg, 0.324 mmol; 14:1 ratio of diene isomers), was azeotropically dried with benzene $(3 \times 0.5 \mathrm{~mL})$ using a double manifold and further dried by heating to $50^{\circ} \mathrm{C}$ under vacuum (0.2 torr) for $24 \mathrm{~h}$. The flask was back-filled with argon, dissolved in 1.5 $\mathrm{mL}$ of freshly distilled THF and cooled to $-78^{\circ} \mathrm{C}$. The resultant solution was treated with methyllithiumlithium bromide complex $(2.2 \mathrm{M}$ in pentane, $0.147 \mathrm{~mL})$, warmed to $0{ }^{\circ} \mathrm{C}$, stirred for $30 \mathrm{~min}$., and then recooled to $-78{ }^{\circ} \mathrm{C}$. To this orange/red solution was transferred dropwise via syringe a solution of aldehyde (-)-C (128 mg, $0.287 \mathrm{mmol})$ in THF $(1.0 \mathrm{~mL}+1 \times 0.5 \mathrm{~mL}$ rinse) over $45 \mathrm{~min}$. The resulting orange solution was stirred for a further $30 \mathrm{~min}$. at $-78{ }^{\circ} \mathrm{C}$, and then warmed to $-10{ }^{\circ} \mathrm{C}$ and allowed to stir for $3 \mathrm{hrs}$. The resulting light yellow solution was quenched with saturated $\mathrm{NH}_{4} \mathrm{Cl}$ and diluted $\left(\mathrm{Et}_{2} \mathrm{O} / \mathrm{H}_{2} \mathrm{O}\right)$. The layers were separated, and the aqueous layer was extracted $\left(3 \times \mathrm{Et}_{2} \mathrm{O}\right)$. The combined organic layers were dried $\left(\mathrm{MgSO}_{4}\right)$, concentrated, and chromatographed (gradient elution: $3 \%$ EtOAc/hexanes $50 \%$ EtOAc/hexanes; then $40 \% \mathrm{CH}_{3} \mathrm{CN} / \mathrm{CH}_{2} \mathrm{Cl}_{2}$ ) to afford cis isomer (+)-15 (164 mg, 48\%; clear oil, $14: 1$ ratio of diene isomers), trans isomer (11.5 mg, $3.4 \%$; clear oil; $14: 1$ ratio of diene isomers), and phosphonium salt $(+)-14$ (132 mg, 39\%; $14: 1$ ratio of diene isomers): Cis olefin $(+)-15:[\square]_{\mathrm{D}}^{23}+32.0^{\circ}(\mathrm{C}$ $\left.0.3 \mathrm{CHCl}_{3}\right)$; IR ( $\left.\mathrm{NaCl}\right) 2958,2929,2884,2857,1734,1472,1253,1045,836 \mathrm{~cm}^{-1} ;{ }^{1} \mathrm{H}-\mathrm{NMR}(500 \mathrm{MHz}$, $\left.\mathrm{CDCl}_{3}\right) \square 7.25(\mathrm{~d}, J=8.1 \mathrm{~Hz}, 2 \mathrm{H}), 6.84(\mathrm{~d}, J=8.9 \mathrm{~Hz}, 2 \mathrm{H}), 6.57$ (ddd, $\left.J=17.1,10.8,10.8 \mathrm{~Hz}, 1 \mathrm{H}\right), 6.00$ (apparent t, $J=10.8 \mathrm{~Hz}, 1 \mathrm{H}$ ), 5.55 (apparent t, $J=10.8 \mathrm{~Hz}, 1 \mathrm{H}$ ), 5.31 (dd, $J=10.8,7.8 \mathrm{~Hz}, 1 \mathrm{H}$ ), 5.24$5.16(\mathrm{~m}, 2 \mathrm{H}), 5.10(\mathrm{~d}, J=10.4 \mathrm{~Hz}, 1 \mathrm{H}), 4.99(\mathrm{~d}, J=10.0 \mathrm{~Hz}, 1 \mathrm{H}), 4.80$ (apparent t, $J=8.9 \mathrm{~Hz}, 1 \mathrm{H}$ ), 4.59 $\left(\mathrm{ABq}, J_{\mathrm{AB}}=6.7 \mathrm{~Hz}, \square_{\mathrm{AB}}=26.4 \mathrm{~Hz}, 2 \mathrm{H}\right), 4.54(\mathrm{~d}, J=10.4 \mathrm{~Hz}, 2 \mathrm{H}), 4.19$ (apparent t, $J=10.8 \mathrm{~Hz}, 1 \mathrm{H}$ ), 3.77 (s, 3H), 3.61 (apparent t, $J=2.6 \mathrm{~Hz}, 1 \mathrm{H}$ ), 3.43 (apparent t, $J=4.1 \mathrm{~Hz}, 1 \mathrm{H}$ ), $3.24(\mathrm{~s}, 3 \mathrm{H}$ ), 3.23 (dd, $J$ = 7.4, 3.7 Hz, 1H), 3.04 (apparent t, $J=5.6 \mathrm{~Hz}, 1 \mathrm{H}$ ), 2.98 (ddd, $J=10.0,6.7,3.3 \mathrm{~Hz}, 1 \mathrm{H}), 2.73-2.65(\mathrm{~m}$, 1H), 2.60 (ddd, $J=15.3,7.4,3.0 \mathrm{~Hz}, 1 \mathrm{H}$ ), 2.49 (ddd, $J=16.8,13.0,6.3 \mathrm{~Hz}, 1 \mathrm{H}$ ), 2.02 (apparent t, $J=$ $12.3 \mathrm{~Hz}, 1 \mathrm{H}), 1.85-1.61(\mathrm{~m}, 4 \mathrm{H}), 1.60-1.54(\mathrm{~m}, 2 \mathrm{H}), 1.54(\mathrm{~s}, 3 \mathrm{H}), 1.22(\mathrm{~d}, J=7.4 \mathrm{~Hz}, 3 \mathrm{H}), 1.08(\mathrm{~d}, J=6.7$ $\mathrm{Hz}, 3 \mathrm{H}), 0.99(\mathrm{~d}, J=7.1 \mathrm{~Hz}, 3 \mathrm{H}), 0.96-0.91(\mathrm{~m}, 6 \mathrm{H}), 0.93(\mathrm{~s}, 9 \mathrm{H}), 0.90(\mathrm{~d}, J=6.7 \mathrm{~Hz}, 3 \mathrm{H}), 0.87(\mathrm{~s}, 9 \mathrm{H})$, 0.85 (s, 9H), 0.70 (d, J = $7.1 \mathrm{~Hz}, 3 \mathrm{H}$ ), 0.08 (s, 3H), 0.07 (s, 3H), 0.06 (s, 3H), 0.05 (s, 3H), 0.03 (br s, 6H); ${ }^{13} \mathrm{C}-\mathrm{NMR}\left(125 \mathrm{MHz}, \mathrm{CDCl}_{3}\right) \square$ 173.3, 158.9, 134.4, 133.6, 132.4, 132.1, 131.9, 131.2, 130.8, 129.1, 129.0, 
117.6, 113.6, 97.4, 86.3, 84.5, 77.0, 74.9, 74.69, 74.68, 64.6, 55.9, 55.2, 43.9, 42.3, 40.1, 36.2, 35.5, 35.3 (2), 34.2, 34.1, 26.2, 25.8, 25.6, 23.0, 18.6, 18.5, 18.0, 17.8, 16.63, 16.61, 16.2, 14.7, 13.9, 10.5, -3.29, $-3.32,-4.4,-4.6,-4.9(2)$; high resolution mass spectrum $(\mathrm{ES}+) \mathrm{m} / \mathrm{z} 1079.7214\left[(\mathrm{M}+\mathrm{Na})^{+}\right.$; calcd for $\left.\mathrm{C}_{60} \mathrm{H}_{108} \mathrm{OgSi} \mathrm{Na}: 1079.7199\right]$.

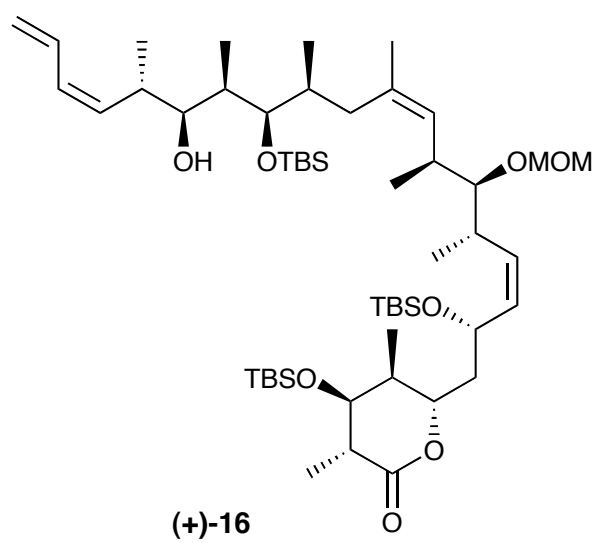

Alcohol (+)-16. At $0{ }^{\circ} \mathrm{C}$, a solution of PMB ether $(+)-15(124 \mathrm{mg}, 0.117 \mathrm{mmol}, 14: 1$ mixture of cis/trans diene isomers) in $\mathrm{CH}_{2} \mathrm{Cl}_{2}(6 \mathrm{~mL})$ was treated with $\mathrm{H}_{2} \mathrm{O}(100 \mathrm{LL})$ and $\mathrm{DDQ}(40 \mathrm{mg}, 0.160 \mathrm{mmol})$. The mixture was stirred for $50 \mathrm{~min}$. at $0{ }^{\circ} \mathrm{C}$, warmed to $\mathrm{rt}$ and stirred an additional $15 \mathrm{~min}$. The mixture was quenched with $7.0 \mathrm{~mL}$ saturated $\mathrm{NaHCO}_{3}$, and diluted with $\mathrm{CH}_{2} \mathrm{Cl}_{2}(25 \mathrm{~mL})$ and $\mathrm{H}_{2} \mathrm{O}(30 \mathrm{~mL})$. The layers were separated and the aqueous layer was extracted with $3 \times 25 \mathrm{~mL} \mathrm{CH}_{2} \mathrm{Cl}_{2}$. The combined organic layers were washed with brine $(10 \mathrm{~mL})$, dried $\left(\mathrm{MgSO}_{4}\right)$, filtered, and concentrated. Flash chromatography (gradient elution; 5\% EtOAc $\square$ 20\% EtOAc/hexanes) provided (+)-16 (101 mg, 92\%) as a colorless oil: $\left.[\square]_{\mathrm{D}}^{23}+35.6^{\circ}\left(c \mathrm{1.0}, \mathrm{CDCl}_{3}\right)\right]$; IR $(\mathrm{NaCl}) 3510,2957,2929,2884,2857,1734,1472,1252,1097,1044$ $\mathrm{cm}^{-1}$; ${ }^{1} \mathrm{H}-\mathrm{NMR}\left(500 \mathrm{MHz}, \mathrm{CDCl}_{3}\right) \square 6.61$ (ddd, $J=17.1,10.9,10.8 \mathrm{~Hz}, 1 \mathrm{H}$ ), 6.12 (apparent $\mathrm{t}, J=11.1 \mathrm{~Hz}$, 1H), 5.34-5.28 (m, 2H), 5.25-5.19 (m, 1H), $5.23(\mathrm{~d}, J=10.1 \mathrm{~Hz}, 1 \mathrm{H}), 5.13(\mathrm{~d}, J=10.1 \mathrm{~Hz}, 1 \mathrm{H}), 5.02(\mathrm{~d}, J$ $=10.1 \mathrm{~Hz}, 1 \mathrm{H}), 4.80$ (apparent $\mathrm{t}, J=8.6 \mathrm{~Hz}, 1 \mathrm{H}), 4.59\left(\mathrm{ABq}, J_{\mathrm{AB}}=6.7 \mathrm{~Hz}, \square_{\mathrm{AB}}=18.9 \mathrm{~Hz}, 2 \mathrm{H}\right), 4.49$, (apparent t, $J=10.1 \mathrm{~Hz}, 1 \mathrm{H}$ ), 3.63 (apparent t, $J=2.6 \mathrm{~Hz}, 1 \mathrm{H}$ ), 3.60 (dd, $J=5.6,3.4 \mathrm{~Hz}, 1 \mathrm{H}$ ), 3.34 (s, 3H), 3.34-3.30 (m, 1H), 3.08 (apparent t, $J=5.6 \mathrm{~Hz}, 1 \mathrm{H}$ ), 2.79 (ddd, $J=16.7,14.1,6.7 \mathrm{~Hz}, 1 \mathrm{H}$ ), 2.69 (ddd, $J=16.0,12.6,6.3 \mathrm{~Hz}, 1 \mathrm{H}$ ), 2.60 (ddd, $J=14.9,7.4,2.6 \mathrm{~Hz}, 1 \mathrm{H}$ ), 2.54 (ddd, $J=16.4,12.6,6.3 \mathrm{~Hz}$, $1 \mathrm{H}), 2.18$ (apparent t, $J=12.3 \mathrm{~Hz}, 1 \mathrm{H}), 1.90-1.75(\mathrm{~m}, 4 \mathrm{H}), 1.73-1.69(\mathrm{~m}, 2 \mathrm{H}), 1.59(\mathrm{~s}, 3 \mathrm{H}), 1.55(\mathrm{~m}, 1 \mathrm{H})$, $1.22(\mathrm{~d}, J=7.4 \mathrm{~Hz}, 3 \mathrm{H}), 0.97-0.92(\mathrm{~m}, 15 \mathrm{H}), 0.91(\mathrm{~s}, 9 \mathrm{H}), 0.87(\mathrm{~s}, 9 \mathrm{H}), 0.85(\mathrm{~s}, 9 \mathrm{H}), 0.74(\mathrm{~d}, J=6.7 \mathrm{~Hz}$, $3 \mathrm{H}$ ), 0.07 (apparent s, 6H), 0.06 (s, 3H), 0.05 (s, 3H), 0.04 (s, 3H), $0.03(\mathrm{~s}, 3 \mathrm{H}) ;{ }^{13} \mathrm{C}-\mathrm{NMR}(125 \mathrm{MHz}$, $\left.\mathrm{CDCl}_{3}\right) \square 173.4,134.6,133.7,132.3,132.0,131.9,130.9,130.7,118.3,97.5,86.3,78.9,77.0,76.2,74.7$, $64.7,55.9,43.9,42.3,38.0,36.5,36.3,35.5,34.9$, 34.3, 34.2, 26.2, 25.8, 25.7, 23.24, 18.4, 18.0, 17.9, 
$17.1,16.8,16.5,16.2,13.9,13.6,9.4,-3.3,-3.8,-4.4,-4.6,-4.9$ (2); high resolution mass spectrum $(\mathrm{ES}+) \mathrm{m} / 2$ 959.6625 [(M+Na) ${ }^{+}$; calcd for $\left.\mathrm{C}_{52} \mathrm{H}_{1000} \mathrm{O}_{8} \mathrm{Si}_{3} \mathrm{Na}: 959.6624\right]$.

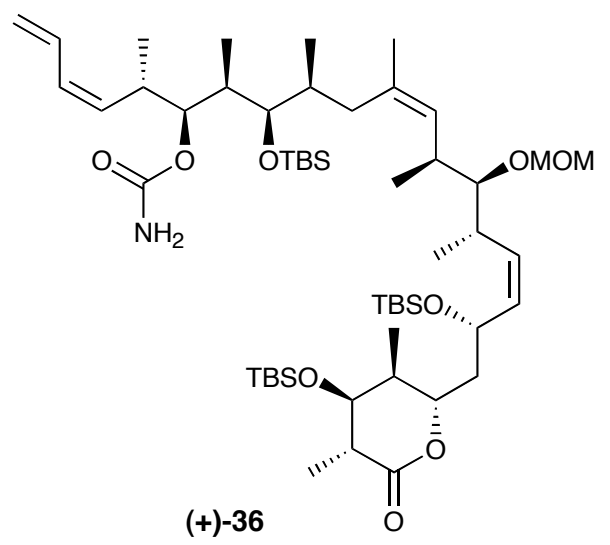

Carbamate (+)-36. A solution of alcohol (+)-16 (10 mg, $0.011 \mathrm{mmol})$ in $\mathrm{CH}_{2} \mathrm{Cl}_{2}(1.0 \mathrm{~mL})$ was treated with a $1 \mathrm{M}$ solution of $\mathrm{Cl}_{3} \mathrm{CCON}=\mathrm{C}=\mathrm{O}$ in toluene $(16 \mathrm{~mL}, 0.016 \mathrm{mmol})$ at room temperature for $30 \mathrm{~min}$. Neutral $\mathrm{Al}_{2} \mathrm{O}_{3}(1 \mathrm{~g})$ was added, followed by $2 \mathrm{~mL} \mathrm{CH}_{2} \mathrm{Cl}_{2}$. After $4 \mathrm{~h}$, the material was transferred to a cottonplugged chromatography column and flushed from the $\mathrm{Al}_{2} \mathrm{O}_{3}$ (EtOAc, $50 \mathrm{~mL}$ ). Concentration and purification by preparatory TLC (250 $\square \mathrm{m}$ plate, $20 \%$ ethyl acetate/hexanes) provided $6.0 \mathrm{mg}(+)-36(60 \%)$ as a colorless oil and $3.5 \mathrm{mg}(35 \%)$ of recovered (+)-16. Carbamate $(+)-36$ : $[\square]_{\mathrm{D}}^{23}+46.2^{\circ}\left(\mathrm{c} 0.5, \mathrm{CHCl}_{3}\right)$; IR ( $\mathrm{NaCl}) 3510,3360,2957,2936,2884,2849,1732,1594,1253,1033 \mathrm{~cm}^{-1} ;{ }^{1} \mathrm{H}-\mathrm{NMR}\left(500 \mathrm{MHz}, \mathrm{CDCl}_{3}\right)$ $\square 6.58$ (ddd, $J=17.1,10.8,10.8 \mathrm{~Hz}, 1 \mathrm{H}$ ), 6.01 (apparent t, $J=11.1 \mathrm{~Hz}, 1 \mathrm{H}$ ), 5.35 (apparent t, $J=10.4 \mathrm{~Hz}$, $1 \mathrm{H}), 5.33-5.27(\mathrm{~m}, 1 \mathrm{H}), 5.25-5.18(\mathrm{~m}, 2 \mathrm{H}), 5.12(\mathrm{~d}, J=10.0 \mathrm{~Hz}, 1 \mathrm{H}), 5.01(\mathrm{~d}, J=10.0 \mathrm{~Hz}, 1 \mathrm{H}), 4.80$ (apparent t, $J=8.9 \mathrm{~Hz}, 1 \mathrm{H}), 4.71$ (apparent $\mathrm{t}, J=6.0 \mathrm{~Hz}, 1 \mathrm{H}), 4.60\left(\mathrm{ABq}, J_{\mathrm{AB}}=6.7 \mathrm{~Hz}, \square_{\mathrm{AB}}=26.4 \mathrm{~Hz}\right.$, 2H), 4.50 (apparent t, $J=10.4 \mathrm{~Hz}, 1 \mathrm{H}$ ), 4.44 (br s, 2H), 3.64 (apparent t, $J=2.4 \mathrm{~Hz}, 1 \mathrm{H}$ ), 3.43 (apparent t, $J=4.1 \mathrm{~Hz}, 1 \mathrm{H}), 3.34(\mathrm{~s}, 3 \mathrm{H}), 3.06$ (apparent $\mathrm{t}, J=5.6 \mathrm{~Hz}, 1 \mathrm{H}), 3.02-2.94(\mathrm{~m}, 1 \mathrm{H}), 2.75-2.66(\mathrm{~m}, 1 \mathrm{H})$, 2.65-2.57 (m, 1H), 2.57-2.47 (m, 1H), 2.12 (apparent $\mathrm{t}, J=12.4 \mathrm{~Hz}, 1 \mathrm{H}), 1.93-1.79(\mathrm{~m}, 3 \mathrm{H}), 1.74-1.66(\mathrm{~m}$, 2H), 1.58 (apparent s, 3H), 1.55-1.50 (m, 1H), $1.23(\mathrm{~d}, J=7.4 \mathrm{~Hz}, 3 \mathrm{H}), 0.98(\mathrm{~d}, J=7.4 \mathrm{~Hz}, 3 \mathrm{H}), 0.96(\mathrm{~d}, J$ $=6.7 \mathrm{~Hz}, 3 \mathrm{H}), 0.94(\mathrm{~d}, J=5.4 \mathrm{~Hz}, 3 \mathrm{H}), 0.94-0.92(\mathrm{~m}, 6 \mathrm{H}), 0.91(\mathrm{~s}, 9 \mathrm{H}), 0.87(\mathrm{~s}, 9 \mathrm{H}), 0.85(\mathrm{~s}, 9 \mathrm{H}), 0.71(\mathrm{~d}$, $J=6.7 \mathrm{~Hz}, 3 \mathrm{H}$ ), 0.08 (s, 3H), 0.06 (apparent s, 9H), $0.04(\mathrm{~s}, 3 \mathrm{H}), 0.03(\mathrm{~s}, 3 \mathrm{H}) ;{ }^{13} \mathrm{C}-\mathrm{NMR}(125 \mathrm{MHz}, \mathrm{MeOH}$ -d6) $\mathrm{l}$ 174.9, 158.7, 133.5, 132.9, 131.9, 131.8, 131.6, 130.5, 129.5, 117.1, 97.3, 86.5, 77.8, 76.7, 74.4, $64.5,55.0,44.3,42.0,38.4,36.4$, 35.6, 34.9, 34.3, 34.2, 33.4, 25.4, 24.9, 18.0, 17.5, 17.4, 16.6, 16.1, $15.7,15.2,13.2,13.1,9.3,-4.4,-4.5,-5.4,-5.9(2),-6.2$; high resolution mass spectrum (ES+) $\mathrm{m} / \mathrm{z}$ 1002.6638 [(M+Na) $)^{+}$; calcd for $\left.\mathrm{C}_{53} \mathrm{H}_{101} \mathrm{NO}_{9} \mathrm{Si}_{3} \mathrm{Na}: 1002.6682\right]$. 


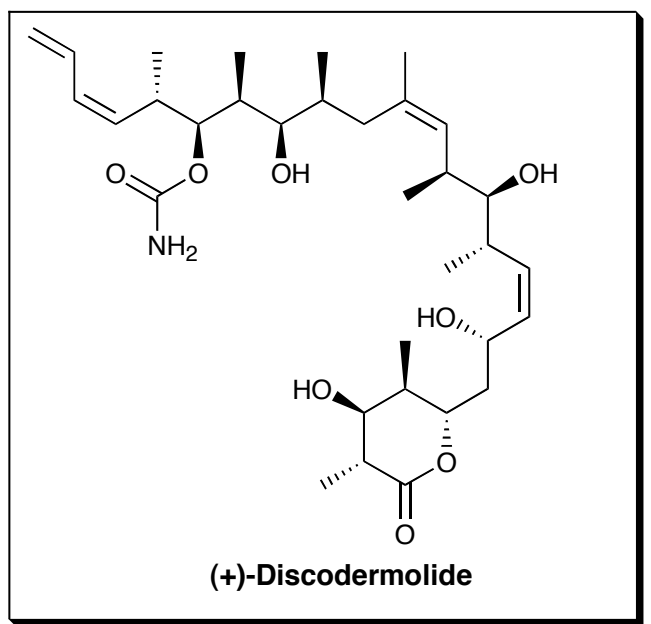

(+)-Discodermolide (1). Carbamate (+)-36 (6.0 mg, $0.006 \mathrm{mmol})$ was dissolved in $\mathrm{MeOH}(1.5 \mathrm{~mL})$ and stirred for $15 \mathrm{~min}$. at room temperature. Aqueous hydrochloric acid $(4 \mathrm{~N}, 1.5 \mathrm{~mL})$ was added in small portions over 4 hours at a rate which minimized precipitation (ca. 10 to $15 \mathrm{~min}$. intervals). After $4 \mathrm{~h}$, an additional $1 \mathrm{~mL}$ of $4 \mathrm{~N}$ aq HCl was added in one portion, and the resulting solution was stirred for $2 \mathrm{~h}$ at $\mathrm{rt}$, diluted with $10 \mathrm{~mL}$ of water and poured into $15 \mathrm{~mL}$ of EtOAc. The resulting mixture was quenched via careful portionwise addition of $\mathrm{NaHCO}_{3}$ until $\mathrm{CO}_{2}$ evolution ceased. The layers were separated, the aqueous layer was saturated with $\mathrm{NaCl}$ and extracted $(3 \times$ EtOAc). The combined organic layers were washed with brine $(1 \times 10 \mathrm{~mL})$, dried $\left(\mathrm{MgSO}_{4}\right)$, filtered, and concentrated. Flash chromatography using washed $\mathrm{SiO}_{2}\left(10 \% \mathrm{MeOH} / \mathrm{CH}_{2} \mathrm{Cl}_{2}\right.$ then $55 \% \mathrm{EtOAc} /$ hexanes) via gradient elution (55\% EtOAc/hexanes then $10 \% \mathrm{MeOH} / \mathrm{CH}_{2} \mathrm{Cl}_{2}$ ) provided (+)-discodermolide $(3.1 \mathrm{mg}, 87 \%$ yield) as a white amorphous solid, whose spectral data ( $\left.{ }^{1} \mathrm{H}-\mathrm{NMR}, \mathrm{HRMS}\right)$ matched that previously reported for the natural product. 